\title{
Fractional Schrödinger dynamics and decoherence
}

\author{
Kay Kirkpatrick* and Yanzhi Zhang ${ }^{\dagger}$
}

\begin{abstract}
We study the dynamics of the Schrödinger equation with a fractional Laplacian $(-\Delta)^{\alpha}$, and the decoherence of the solution is observed. Analytically, we obtain equations of motion for the expected position and momentum in the fractional Schödinger equation, equations that are the fractional counterpart of the well-known Newtonian equations of motion for the standard $(\alpha=1)$ Schrödinger equation. Numerically, we propose an explicit, effective numerical method for solving the time-dependent fractional nonlinear Schrödinger equation-a method that has high order spatial accuracy, requires little memory, and has low computational cost. We apply our method to study the dynamics of fractional Schrödinger equation and find that the nonlocal interactions from the fractional Laplacian introduce decoherence into the solution. The local nonlinear interactions can however reduce or delay the emergence of decoherence. Moreover, we find that the solution of the standard NLS behaves more like a particle, but the solution of the fractional NLS behaves more like a wave with interference effects.
\end{abstract}

Keywords. Fractional Schrödinger equation, Fourier pseudo-spectral method, center of mass, fractional momentum, decoherence.

\section{Introduction}

Dispersive equations with nonlocal operators like the fractional Laplacian are of great interest: fractional Schrödinger equations arise in physical systems where particles interact with one another over long distances [1-4], especially in models of charge transport in large-scale organic polymers such as DNA [5]. A special case, the square root of the Laplacian, can be viewed as a model for pseudo-relativistic Boson stars [6,7]. These fractional Schrödinger equations have been studied in the physics literature $[1,5]$, with heuristic arguments justifying the derivation of these nonlocal continuum dynamics from the underlying biophysics, which is understood to be modeled by a discrete nonlinear Schrödinger equation (NLS), with interactions (e.g., between different base pairs in a strand of DNA) that decay like inverse power laws. Recently, this heuristic derivation has been rigorously justified for classes of fractional NLS equations, that they arise from microscopic (or properly speaking, mesoscopic) lattice systems with long-range interactions when passing to the continuum limit [8].

We consider the dimensionless fractional NLS equation $[2,9,10]$ :

$$
\begin{aligned}
& i \frac{\partial \psi(\mathbf{x}, t)}{\partial t}=\left(\frac{1}{2}(-\Delta)^{\alpha}+V(\mathbf{x})+\beta|\psi(\mathbf{x}, t)|^{2}\right) \psi(\mathbf{x}, t), \quad \mathbf{x} \in \mathbb{R}^{d}, \quad t>0, \\
& \psi(\mathbf{x}, 0)=\psi_{0}(\mathbf{x}), \quad \mathbf{x} \in \mathbb{R}^{d},
\end{aligned}
$$

${ }^{*}$ University of Illinois at Urbana-Champaign, Department of Mathematics, 1409 W. Green Street, Urbana, IL 61801 (Email: kkirkpat@illinois.edu)

${ }^{\dagger}$ Department of Mathematics and Statistics, Missouri University of Science and Technology, Rolla, MO 65409 (Email: zhangyanz@mst.edu) 
where $\psi(\mathbf{x}, t)$ is a complex-valued wave function of spatial coordinate vector $\mathbf{x} \in \mathbb{R}^{d}(d=1,2$, or 3$)$ and time $t \geq 0$. The constant $\beta \in \mathbb{R}$ describes the strength of local interactions, and the interactions are repulsive or defocusing (resp. attractive or focusing) if $\beta>0$ (resp. $\beta<0$ ). The real-valued function $V(\mathbf{x})$ represents an external trapping potential. In this paper, we consider a harmonic potential of the form: $V(\mathbf{x})=\frac{1}{2} \mathbf{x}^{T} \Lambda \mathbf{x}$; the diagonal matrix $\Lambda=\gamma_{x}^{2}$ if $d=1, \Lambda=\operatorname{diag}\left(\gamma_{x}^{2}, \gamma_{y}^{2}\right)$ if $d=2$, and $\Lambda=\operatorname{diag}\left(\gamma_{x}^{2}, \gamma_{y}^{2}, \gamma_{z}^{2}\right)$ if $d=3$, with $\gamma_{x}, \gamma_{y}$ and $\gamma_{z}$ the dimensionless trapping frequencies in $x^{-}, y$, and $z$-direction, respectively. For $\alpha>0$, the fractional Laplacian $(-\Delta)^{\alpha}$ is defined by $[11,12]$

$$
(-\Delta)^{\alpha} \psi(\mathbf{x}, t):=\mathcal{F}^{-1}\left[|\xi|^{2 \alpha} \mathcal{F}(\psi)\right], \quad \mathbf{x} \in \mathbb{R}^{d},
$$

where $\mathcal{F}$ defines the Fourier transform, i.e.,

$$
\mathcal{F}(\psi)(\xi, t)=\int_{\mathbb{R}^{d}} \psi(\mathbf{x}, t) e^{-i \xi \cdot \mathbf{x}} d \mathbf{x}
$$

and $\mathcal{F}^{-1}$ represents the inverse Fourier transform. If $\alpha=1,(1.1)$ becomes the standard (nonfractional) NLS equation, also known as the Gross-Pitaevskii equation (GPE) in the literature of Bose-Einstein condensation [13-15]. The case $\alpha=1 / 2$ is interesting because it corresponds to the Hilbert-NLS and is challenging because the dispersion relation $\omega(k)=-|k|$ is like the water wave dispersion. In this paper, we are interested in the case $1 / 2<\alpha \leq 1$, with dispersion relation $\omega(k)=-|k|^{2 \alpha}$ between the wave and Schrödinger cases, and will leave the case of $\alpha=1 / 2$ for future study.

The general fractional NLS (1.1) has some conserved quantities for $t \geq 0$ : the $L^{2}{ }^{-}$norm, or mass of the wave function, which we will take to be normalized,

$$
\|\psi(\cdot, t)\|^{2}:=\int_{\mathbb{R}^{d}}|\psi(\mathbf{x}, t)|^{2} d \mathbf{x}=\int_{\mathbb{R}^{d}}|\psi(\mathbf{x}, 0)|^{2} d \mathbf{x}=1
$$

and the total energy,

$$
E(t):=\int_{\mathbb{R}^{d}}\left[\frac{1}{2}\left|\nabla^{\alpha} \psi\right|^{2}+V(\mathbf{x})|\psi|^{2}+\frac{\beta}{2}|\psi|^{4}\right] d \mathbf{x}=E(0),
$$

where we write the operator $\nabla^{s}=-(-\Delta)^{s / 2}$, for $s>0$. These conserved quantities can be used as benchmarks in analysis and simulation of the fractional NLS.

Existence and uniqueness of ground states of the elliptic problem associated with the fractional NLS have been studied in one dimension [16] and higher dimensions in the radial case [17]. By contrast, the time-evolution of initial states for the fractional NLS is not well understood qualitatively, though there are abstract well-posedness results in the literature $[8,18,19]$ and finite-time blow-up results for closely related equations $[7,20]$. Recently, soliton dynamics have been studied for the fractional NLS in the semi-classical limit, and found to concentrate along a trajectory determined by the Newtonian type equation [9]. However, the fractional counterpart of the NLS equations of motion for expected position and momentum were thought hard to obtain [9, Remark 4.7], equations of motion that we will present in Theorem 2.1. Additionally, numerical investigations on the fractional NLS dynamics in the literature remain scant.

In this paper, we analytically and numerically study the solution dynamics of the fractional NLS in comparison with the standard NLS, so as to understand the nonlocal effects of the fractional Laplacian on the dynamics. We study solutions of the fractional linear and nonlinear Schrödinger equations in the presence of an external harmonic potential, and we observe conditions under which 
there is a loss of the ground state profile when it evolves additional peak(s), and conditions under which there is emergence of high frequencies. The loss of the ground state profile in the fractional NLS does not appear to arise from the nonlinearity, but from the nonlocal interactions of the fractional Laplacian, possibly from its interaction with the potential, especially if the initial state is not symmetric with respect to the center of the external potential. In fact, our simulations suggest that the nonlinearity helps to maintain the shape of the ground state profile, delaying or reducing the leakage to high frequencies, albeit with some fluctuations. Our main contributions in this paper include:

(i) We obtain the equations of motion for expected positions and momentum for the fractional Schrödinger equation, extending the results in the literature [9]. We show that the equations of motion for the fractional NLS do not form a closed system, and they also depend on the nonlinearity, which is different from the well-known Newtonian equations of motion for the standard NLS.

(ii) We propose a time-splitting Fourier pseudo-spectral method for simulating the dynamics of fractional NLS. It has the spectral-order accuracy in space and the second-order accuracy in time. One main merit of our method is that it requires low memory and computational costs, independent of the fractional power $\alpha$.

(iii) We numerically study the dynamics of the fractional Schrödinger equation with a harmonic potential in both linear and nonlinear cases, examine the loss of the ground state profile that is associated to the nonlocal interactions of the fractional Laplacian rather than the nonlinear term.

\section{Equations of motion for expected position and momentum}

For the standard (non-fractional) Schrödinger equation, the Ehrenfest theorem gives Newtonian equations of motion for the expectations of position and momentum observables, equations that are closed and have periodic solutions [21]. For example, in the traditional nonlinear Schrödinger equation, Ehrenfest-type results have been proved [22], and in the semiclassical limit of the nonlinear Schrödinger equation, the center of mass (expected position) is also known to converge to the solution of a Newtonian equation [23,24]. However, the fractional counterpart of the NLS equations of motion for expected position and momentum still remains open (see [9, Remark 4.7]). In this section, we obtain the analogous equations for the fractional Schrödinger equation and show that they are not closed and have a fractional momentum differing from the usual momentum.

The center of mass $\langle X\rangle$ is the expected value of the position operator $X[\psi(\mathbf{x}, t)]:=\mathbf{x} \psi(\mathbf{x}, t)$, which can be written using the Hilbert space inner product $\langle\cdot, \cdot\rangle$ or explicitly as an integral:

$$
\langle X\rangle:=\langle\psi, X \psi\rangle=\int_{\mathbb{R}^{d}} \mathbf{x}|\psi(\mathbf{x}, t)|^{2} d \mathbf{x}, \quad t \geq 0 .
$$

Following [2], we define the fractional momentum operator:

$$
P_{\alpha}:=-i \alpha \nabla^{2 \alpha-1}=\alpha\left|P^{2}\right|^{\alpha-1} P, \quad \text { for } \quad \frac{1}{2}<\alpha \leq 1,
$$

where $P=-i \nabla$ is the usual momentum operator. The operator $\nabla^{2 \alpha-k}$, for positive integer $k$, can be defined by composing $\nabla^{2 \alpha-k}:=\nabla^{2 \alpha} \nabla^{-k}$, where the $k$-th order integral operator $\nabla^{-k}$ is defined, 
for instance, through Cauchy's formula for iterated integrals in one dimension (here $a$ is arbitrary and fixed):

$$
\nabla^{-k} f(x)=f^{(-k)}(x)=\frac{1}{(k-1) !} \int_{a}^{x}(x-y)^{k-1} f(y) d y .
$$

It is easy to see that the Fourier multiplier of the fractional momentum operator $P_{\alpha}$ is $i \alpha \xi|\xi|^{2 \alpha-2}$. Then the expected fractional momentum is defined as

$$
\left\langle P_{\alpha}\right\rangle:=\left\langle\psi, P_{\alpha} \psi\right\rangle=-i \alpha \int_{\mathbb{R}^{d}} \psi^{*} \nabla^{2 \alpha-1} \psi d \mathbf{x}, \quad t \geq 0, \quad \text { for } \frac{1}{2}<\alpha \leq 1,
$$

where $\psi^{*}$ represents the complex conjugate of the function $\psi$. The following theorem establishes the time evolution of the expected position and fractional momentum (addressing the open problem in $[9$, p. 20]).

Theorem 2.1. For a solution $\psi=\psi(\mathbf{x}, t)$ of the fractional $N L S$ (1.1) with harmonic potential and $\alpha \in\left(\frac{1}{2}, 1\right]$, we have the following equations of motion for $t>0$ :

$$
\begin{aligned}
\frac{d}{d t}\langle X\rangle & =\left\langle P_{\alpha}\right\rangle, \\
\frac{d}{d t}\left\langle P_{\alpha}\right\rangle & =\left\langle W_{\alpha}\right\rangle,
\end{aligned}
$$

where the quantity $\left\langle W_{\alpha}\right\rangle$ is the expectation of an operator and can be defined by:

$$
\begin{aligned}
\left\langle W_{\alpha}\right\rangle:= & \alpha(2 \alpha-1)\left\langle(-\nabla V)\left|P^{2}\right|^{\alpha-1}\right\rangle-\alpha(\alpha-1)(2 \alpha-1)\left\langle\left(\nabla^{2} V\right) \nabla^{2 \alpha-3}\right\rangle \\
& -\alpha \beta \sum_{j \geq 1}\left(\begin{array}{c}
2 \alpha-1 \\
j
\end{array}\right)\left\langle\psi,\left(\nabla^{2 \alpha-1-j} \psi\right)\left(\nabla^{j}\left(|\psi|^{2}\right)\right)\right\rangle .
\end{aligned}
$$

Proof. The time derivative of the center of mass can be computed:

$$
\begin{aligned}
\frac{d}{d t}\langle X\rangle= & \left\langle\psi_{t}, \mathbf{x} \psi\right\rangle+\left\langle\psi, \mathbf{x} \psi_{t}\right\rangle \\
= & \left\langle-\frac{i}{2}(-\Delta)^{\alpha} \psi-i V \psi-i \beta|\psi|^{2} \psi, \mathbf{x} \psi\right\rangle \\
& +\left\langle\psi,-\frac{i}{2} \mathbf{x}(-\Delta)^{\alpha} \psi-i \mathbf{x} V \psi-i \beta \mathbf{x}|\psi|^{2} \psi\right\rangle \\
= & \frac{i}{2}\left\langle\psi,(-\Delta)^{\alpha} \mathbf{x} \psi-\mathbf{x}(-\Delta)^{\alpha} \psi\right\rangle .
\end{aligned}
$$

Then using the fractional version of the Leibniz rule [12], this becomes:

$$
\begin{aligned}
\frac{d}{d t}\langle X\rangle & =\frac{i}{2}\left\langle\psi,-\sum_{j=0}^{\infty}\left(\begin{array}{c}
2 \alpha \\
j
\end{array}\right)\left(\nabla^{2 \alpha-j} \psi\right)\left(\nabla^{j} \mathbf{x}\right)+\mathbf{x} \nabla^{2 \alpha} \psi\right\rangle \\
& =\left\langle\psi,-i \alpha \nabla^{2 \alpha-1} \psi\right\rangle=\left\langle P_{\alpha}\right\rangle .
\end{aligned}
$$

To compute the time derivative of the expected fractional momentum, we split it into three terms, one for each of the three terms in the fractional NLS (1.1): kinetic, external potential, and nonlinear.

$$
\begin{aligned}
\frac{d}{d t}\left\langle P_{\alpha}\right\rangle & =\left\langle\psi_{t}, \alpha\left|P^{2}\right|^{\alpha-1} P \psi\right\rangle+\left\langle\psi, \alpha\left|P^{2}\right|^{\alpha-1} P \psi_{t}\right\rangle \\
& =\left\langle i \psi_{t}, \alpha\left|P^{2}\right|^{\alpha-1} \nabla \psi\right\rangle+\left\langle\psi, \alpha\left|P^{2}\right|^{\alpha-1}(-\nabla) i \psi_{t}\right\rangle \\
& =I+I I+I I I
\end{aligned}
$$


where the terms $I, I I$, and $I I I$ are defined using the fractional NLS (1.1) by:

$$
\begin{aligned}
I & =\left\langle\frac{1}{2}(-\Delta)^{\alpha} \psi, \alpha\left|P^{2}\right|^{\alpha-1} \nabla \psi\right\rangle+\left\langle\psi, \alpha\left|P^{2}\right|^{\alpha-1}(-\nabla) \frac{1}{2}(-\Delta)^{\alpha} \psi\right\rangle, \\
I I & =\left\langle V \psi, \alpha\left|P^{2}\right|^{\alpha-1} \nabla \psi\right\rangle+\left\langle\psi, \alpha\left|P^{2}\right|^{\alpha-1}(-\nabla)(V \psi)\right\rangle, \\
I I I & =\left\langle\beta|\psi|^{2} \psi, \alpha\left|P^{2}\right|^{\alpha-1} \nabla \psi\right\rangle+\left\langle\psi, \alpha\left|P^{2}\right|^{\alpha-1}(-\nabla)\left(\beta|\psi|^{2} \psi\right)\right\rangle .
\end{aligned}
$$

Term $I$ vanishes, because it comes from the kinetic part of the Hamiltonian, commutativity of the differential operators, and self-adjointness of the fractional Laplacian:

$$
\begin{aligned}
I & =\left\langle\frac{1}{2}(-\Delta)^{\alpha} \psi, \alpha\left|P^{2}\right|^{\alpha-1} \nabla \psi\right\rangle+\left\langle\psi, \alpha\left|P^{2}\right|^{\alpha-1}(-\nabla) \frac{1}{2}(-\Delta)^{\alpha} \psi\right\rangle \\
& =\left\langle\frac{1}{2}(-\Delta)^{\alpha} \psi, \alpha\left|P^{2}\right|^{\alpha-1} \nabla \psi\right\rangle+\left\langle\frac{1}{2}(-\Delta)^{\alpha} \psi, \alpha\left|P^{2}\right|^{\alpha-1}(-\nabla) \psi\right\rangle=0 .
\end{aligned}
$$

Term $I I$ comes from the external potential, and we rewrite it using the commutator $[A, B]=$ $A B-B A$ :

$$
\begin{aligned}
I I & =\left\langle V \psi, \alpha\left|P^{2}\right|^{\alpha-1} \nabla \psi\right\rangle+\left\langle\psi, \alpha\left|P^{2}\right|^{\alpha-1}(-\nabla)(V \psi)\right\rangle \\
& =\left\langle\psi, V \alpha\left|P^{2}\right|^{\alpha-1} \nabla \psi\right\rangle-\left\langle\psi, \alpha\left|P^{2}\right|^{\alpha-1} \nabla(V \psi)\right\rangle \\
& =\left\langle\psi,\left[V, \alpha\left|P^{2}\right|^{\alpha-1} \nabla\right] \psi\right\rangle .
\end{aligned}
$$

Then by the fractional Leibniz rule on the second term in the commutator, the $j=0$ term canceling with the first term in the commutator, and $V$ being harmonic, only the $j=1$ and $j=2$ terms survive:

$$
\begin{aligned}
I I & =\left\langle\psi, V \alpha\left|P^{2}\right|^{\alpha-1} \nabla \psi\right\rangle-\left\langle\psi, \alpha \sum_{j=0}^{\infty}\left(\begin{array}{c}
2 \alpha-1 \\
j
\end{array}\right)\left(\nabla^{2 \alpha-1-j} \psi\right)\left(\nabla^{j} V\right)\right\rangle \\
& =\left\langle\psi, \alpha\left(\begin{array}{c}
2 \alpha-1 \\
1
\end{array}\right)\left(\nabla^{2 \alpha-2} \psi\right)(-\nabla V)-\alpha\left(\begin{array}{c}
2 \alpha-1 \\
2
\end{array}\right)\left(\nabla^{2 \alpha-3} \psi\right)\left(\nabla^{2} V\right)\right\rangle \\
& =\alpha(2 \alpha-1)\left\langle\psi,(-\nabla V)\left|P^{2}\right|^{\alpha-1} \psi\right\rangle-\alpha(\alpha-1)(2 \alpha-1)\left\langle\psi,\left(\nabla^{2} V\right) \nabla^{2 \alpha-3} \psi\right\rangle .
\end{aligned}
$$

This fractional Leibniz rule corresponds to the choice of the Riesz fractional derivative in the beginning; a different choice of fractional derivative or pseudo-differential calculus would result in a different Leibniz rule with lower-order correction terms.

The last term $I I I$ in the derivative of the momentum (2.6) comes from the nonlinearity:

$$
\begin{aligned}
I I I & =\left\langle\beta|\psi|^{2} \psi, \alpha\left|P^{2}\right|^{\alpha-1} \nabla \psi\right\rangle+\left\langle\psi, \alpha\left|P^{2}\right|^{\alpha-1}(-\nabla)\left(\beta|\psi|^{2} \psi\right)\right\rangle \\
& =\alpha \beta\left\langle\psi,\left[|\psi|^{2},\left|P^{2}\right|^{\alpha-1} \nabla\right] \psi\right\rangle .
\end{aligned}
$$

The second term of the commutator can be expanded using the fractional Leibniz formula, and the $j=0$ term cancels with the first term of the commutator, giving:

$$
I I I=-\alpha \beta \sum_{j \geq 1}\left(\begin{array}{c}
2 \alpha-1 \\
j
\end{array}\right)\left\langle\psi,\left(\nabla^{2 \alpha-1-j} \psi\right)\left(\nabla^{j}\left(|\psi|^{2}\right)\right)\right\rangle .
$$

Putting the three terms together, we get the equation of motion (2.4) and (2.5). 
Remark 2.1. The threshold $\alpha=1 / 2$ is an important one in the study of symmetric stable processes whose infinitesimal generator is the fractional Laplacian: for instance, if $\alpha>1 / 2$ the mean of the stable distribution is well-defined (equal to the location parameter $\mu$ ), but if $\alpha<1 / 2$ the mean of the stable distribution is undefined. This threshold is reflected in the signs of the coefficients in $\left\langle W_{\alpha}\right\rangle$, which are nonnegative if $\alpha \in(1 / 2,1]$.

Remark 2.2. If $V(\mathbf{x})$ is nonzero and not harmonic, then there may be an infinite series in $\left\langle W_{\alpha}\right\rangle$. But, if there is no external potential, i.e., $V(\mathbf{x})=0,\left\langle W_{\alpha}\right\rangle$ has only the last term III in it, i.e.,

$$
\left\langle W_{\alpha}\right\rangle=-\alpha \beta \sum_{j \geq 1}\left(\begin{array}{c}
2 \alpha-1 \\
j
\end{array}\right)\left\langle\psi,\left(\nabla^{2 \alpha-1-j} \psi\right)\left(\nabla^{j}\left(|\psi|^{2}\right)\right)\right\rangle .
$$

Additionally, if there is no nonlinearity, then $\left\langle W_{\alpha}\right\rangle=0$ as in the non-fractional case.

Remark 2.3. If $\alpha=1$, then integration by parts shows that term III in (2.6) is zero, and the operator is simply $W_{\alpha}=-\nabla V=-\Lambda \mathbf{x}$, a multiplication operator. Thus the equations of motion (2.3)-(2.4) reduce to:

$$
\begin{aligned}
\frac{d}{d t}\langle X\rangle & =\langle P\rangle, \\
\frac{d}{d t}\langle P\rangle & =-\Lambda\langle X\rangle,
\end{aligned}
$$

where $\Lambda$ is the diagonal matrix of trapping frequencies of a harmonic potential.

Remark 2.3 shows that when $\alpha=1$, the equations of motion (2.7)-(2.8) form a closed system, independent of the nonlinear parameter $\beta$, and their solution is periodic with period depending on the trapping frequency of the harmonic potential. By contrast, Theorem 2.1 shows that the equations of motion in the fractional $(\alpha<1)$ case are not closed, which is a difference between the fractional and standard NLS dynamics. In the following, we present a numerical illustration of this difference.

Figure 1 displays the time evolution of $\langle X\rangle$ and $\langle P\rangle$ in the one-dimensional (1D) standard $(\alpha=1)$ Schrödinger equation (1.1) with harmonic potential $V(x)=\frac{1}{2} x^{2}$, obtained by numerically simulating (1.1) with the initial condition $\psi_{0}(x)$ as given in (4.1). It shows that both $\langle X\rangle$ and
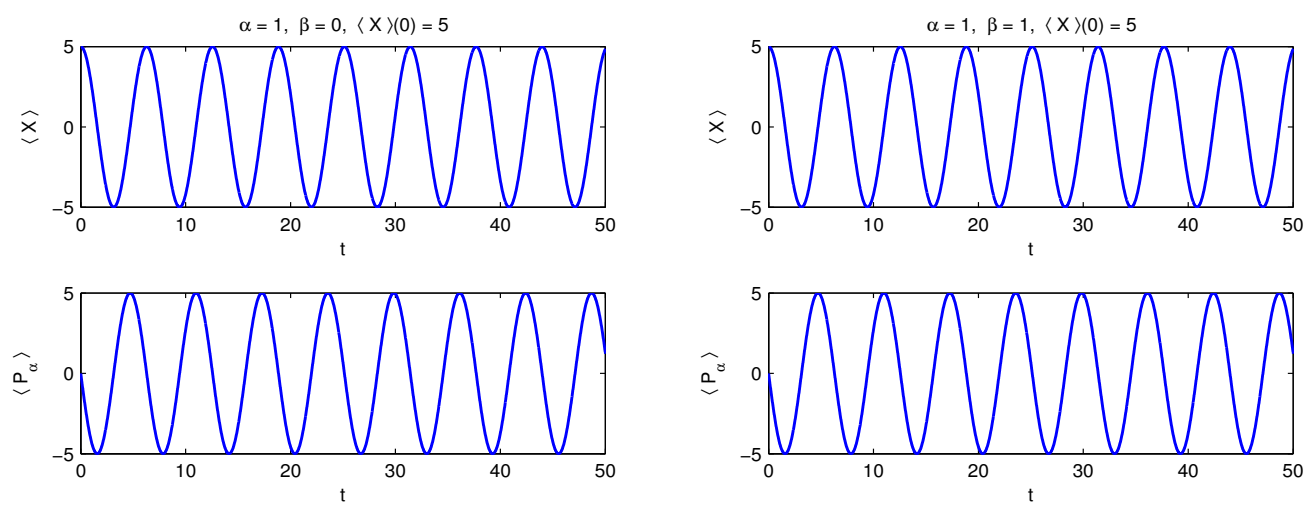

Figure 1: Oscillation of $\langle X\rangle$ and $\langle P\rangle$ in the 1D standard $(\alpha=1)$ Schrödinger equation with harmonic potential $V(x)=\frac{1}{2} x^{2}$, obtained by numerically simulating (1.1) with (4.1).

$\langle P\rangle$ oscillate periodically with period $T=2 \pi$, and their evolution is independent of $\beta$ and $\langle X\rangle(0)$. 
This observation is consistent with our analytical results in Remark 2.3 and those reported in the literature $[14,15,25-27]$.

By contrast, the dynamics of $\langle X\rangle$ and $\left\langle P_{\alpha}\right\rangle$ for the fractional NLS are more complicated (see Figure 2), depending not only on $\alpha$ but also on $\langle X\rangle(0)$ and $\beta$. Decay of $\langle X\rangle$ and $\left\langle P_{\alpha}\right\rangle$ is observed
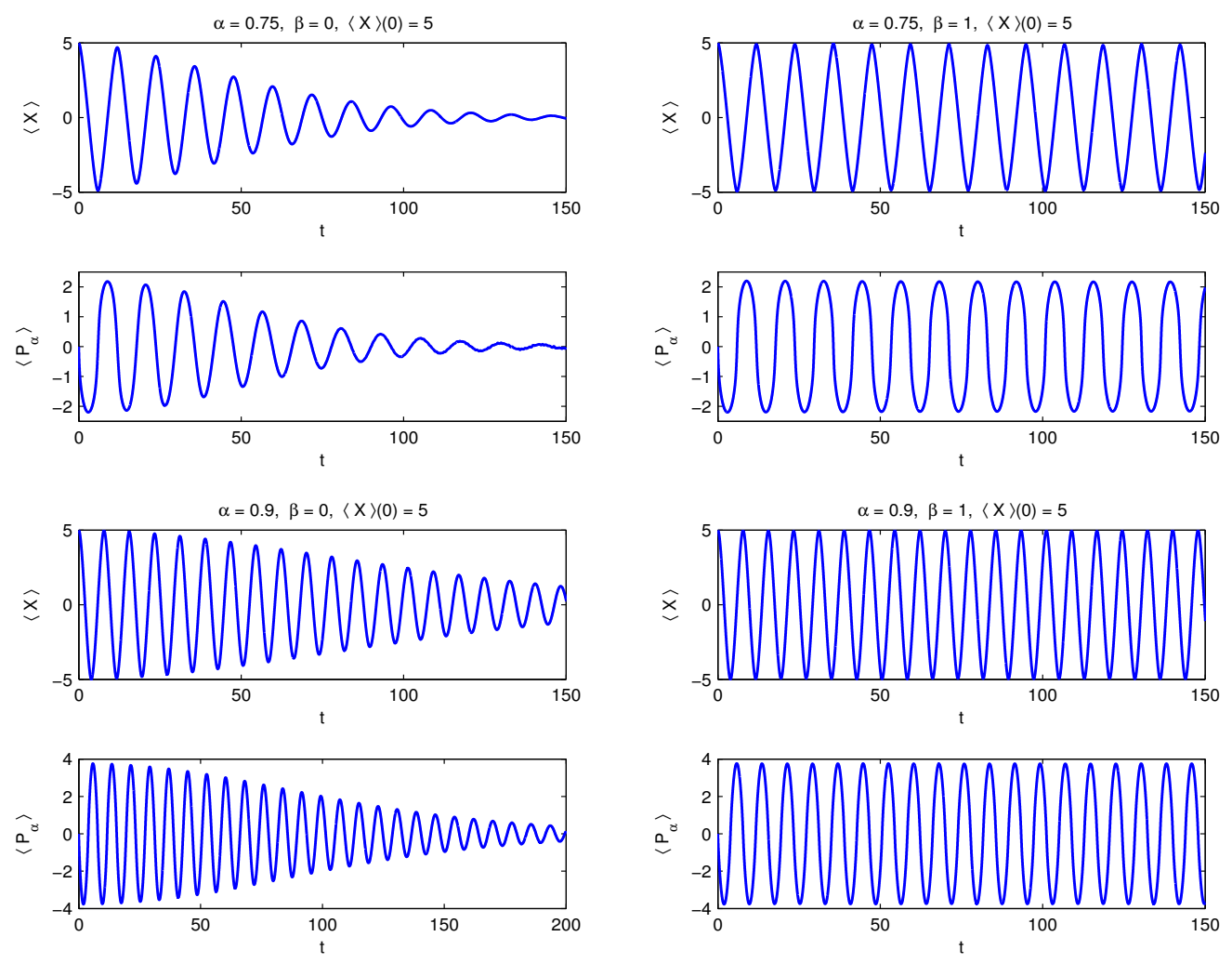

Figure 2: Oscillation and decay of the time evolution of $\langle X\rangle$ and $\left\langle P_{\alpha}\right\rangle$ in the 1D fractional Schrödinger equation (1.1) with harmonic potential $V(x)=\frac{1}{2} x^{2}$, obtained by numerically simulating (1.1) with the initial state (4.1). There is complexity in the solutions that is not apparent in this figure, complexity including decoherence and emergence of high frequencies (discussed in Section 4) even in the linear cases (left).

along with oscillation, especially when $\beta=0$; including the nonlinear interactions can reduce the decay of $\langle X\rangle$ and $\left\langle P_{\alpha}\right\rangle$ during the dynamics. However, the time evolution of $\langle X\rangle$ and $\left\langle P_{\alpha}\right\rangle$ fails to show the decoherence and emergence of high frequencies in the dynamics of fractional NLS; see more discussion in Section 4.

\section{$3 \quad$ Time-splitting Fourier pseudo-spectral method}

There have been numerous studies on fractional differential equations, and various numerical methods have been proposed to solve fractional-in-space diffusion equations; see [28-33] and references therein. Most of these methods are finite difference or finite element methods, which usually have low-order spatial accuracy, and at each time step, they result in solving linear systems of the form $A \mathbf{u}=\mathbf{b}$. However, due to its nonlocality, the discretization of the fractional Laplacian $(-\Delta)^{\alpha}$ yields a full matrix $A$, and thus solving the linear system $A \mathbf{u}=\mathbf{b}$ could be costly. Additionally, the storage of $A$ could be challenging, especially in high spatial dimensions (e.g., $d=2$ or 3 ). Recently, 
a Fourier spectral method was presented in [34] to solve the fractional linear reaction-diffusion equation, and collocation methods based on little sinc functions were proposed in [35] to compute solutions of the fractional linear Schrödinger equation. Both of these new methods have spectral accuracy in space, in contrast to the finite difference and finite element methods. All the above methods, however, are for solving linear equations.

For nonlinear equations, Fourier spectral type methods were proposed in [36-38] to solve the nonlinear dispersive equations, in which the temporal discretization is realized by the integrating factor method or the fourth-order Runge-Kutta method. These methods are conditionally stable, and moreover they are difficult to be applied to solve the fractional NLS with external trapping potential. In this paper, we propose a time-splitting Fourier pseudo-spectral method to solve the time-dependent fractional NLS (1.1)-(1.2), with the following merits: (i) It has spectral-order accuracy in space and second-order accuracy in time. In addition, the temporal accuracy can be easily improved by using a higher-order splitting method. (ii) The method is explicit and straightforward to implement, requiring much less memory and computational costs than finite difference/element methods. (iii) The generalization of our method for solving one-dimensional (1D) fractional NLS to higher spatial dimensions is straightforward. (iv) Our method can be used to solve not only fractional NLS but also the traditional non-fractional NLS $(\alpha=1)$.

First, we truncate (1.1)-(1.2) into a sufficiently large bounded computational domain $\Omega \in \mathbb{R}^{d}$, and without loss of generality, we consider the following problem:

$$
\begin{aligned}
& i \partial_{t} \psi(\mathbf{x}, t)=c(-\Delta)^{\alpha} \psi+V(\mathbf{x}) \psi+\beta|\psi|^{2 \sigma} \psi, \quad \mathbf{x} \in \Omega, \quad t>0, \\
& \psi(\mathbf{x}, 0)=\psi_{0}(\mathbf{x}), \quad \mathbf{x} \in \bar{\Omega},
\end{aligned}
$$

with constants $c>0$ and $\sigma>0$. The fractional NLS in (1.1) corresponds to choosing $c=\frac{1}{2}$ and $\sigma=1$ in (3.1). Here, we consider periodic boundary conditions for the problem (3.1)-(3.2). The use of other boundary conditions for the nonlocal (fractional) equation is a topic that remains to be examined $[39,40]$.

Choose a time step $\tau>0$ and define a time sequence $t_{n}=n \tau$ for $n=0,1, \ldots$. From time $t=t_{n}$ to $t=t_{n+1}$, we solve (3.1) in two splitting steps, i.e., solving:

$$
\begin{aligned}
& i \partial_{t} \psi(\mathbf{x}, t)=V(\mathbf{x}) \psi+\beta|\psi|^{2 \sigma} \psi, \\
& i \partial_{t} \psi(\mathbf{x}, t)=c(-\Delta)^{\alpha} \psi .
\end{aligned}
$$

Multiplying (3.3) by $\psi^{*}$ and then subtracting it from its complex conjugate, we obtain $\partial_{t}\left(|\psi(\mathbf{x}, t)|^{2}\right)=$ 0 , which implies that $|\psi(\mathbf{x}, t)|$ is time invariant on $\left[t_{n}, t_{n+1}\right]$, i.e., $|\psi(\mathbf{x}, t)|=\left|\psi\left(\mathbf{x}, t_{n}\right)\right|$ for $t \in$ $\left[t_{n}, t_{n+1}\right]$. Consequently, for $t_{n} \leq t \leq t_{n+1}$ we can write $(3.3)$ as

$$
i \partial_{t} \psi(\mathbf{x}, t)=\left[V(\mathbf{x})+\beta\left|\psi\left(\mathbf{x}, t_{n}\right)\right|^{2 \sigma}\right] \psi(\mathbf{x}, t),
$$

an equation that is linear in $\psi(\mathbf{x}, t)$. Integrating (3.5) in time gives the solution to (3.3):

$$
\psi(\mathbf{x}, t)=\psi\left(\mathbf{x}, t_{n}\right) e^{-i\left(V(\mathbf{x})+\beta\left|\psi\left(\mathbf{x}, t_{n}\right)\right|^{2 \sigma}\right)\left(t-t_{n}\right)}, \quad t_{n} \leq t \leq t_{n+1} .
$$

Due to the definition of the fractional Laplacian in (1.3), it is natural to use the Fourier pseudospectral method for the spatial discretization of (3.4) [34,41-43]. For simplicity, we present the discretization of (3.4) in one dimension, though the generalization to higher dimension is straightforward. Let $\Omega=(a, b)$ be the $1 \mathrm{D}$ computational domain and $J$ be a positive even integer. Define the mesh size $h=(b-a) / J$ and grid points $x_{j}=a+j h$ for $0 \leq j \leq J$. We assume the approximate ansatz

$$
\psi(x, t)=\sum_{l=-J / 2}^{J / 2-1} \widehat{\psi}_{l}(t) e^{i \mu_{l}(x-a)},
$$


where $\widehat{\psi}_{l}(t)$ represents the $l$-th mode of the Fourier transform of $\psi(x, t)$, and

$$
\mu_{l}=\frac{2 l \pi}{b-a}, \quad-\frac{J}{2} \leq l \leq \frac{J}{2}-1 .
$$

Substituting (3.7) into (3.4) and using the orthogonality of the Fourier basis functions, we obtain

$$
i \frac{d \widehat{\psi}_{l}(t)}{d t}=c\left|\mu_{l}\right|^{2 \alpha} \widehat{\psi}_{l}(t), \quad t_{n} \leq t \leq t_{n+1}, \quad-\frac{J}{2} \leq l \leq \frac{J}{2}-1 .
$$

Integrating it in time, we get

$$
\widehat{\psi}_{l}(t)=\widehat{\psi}_{l}\left(t_{n}\right) e^{-i c\left|\mu_{l}\right|^{2 \alpha}\left(t-t_{n}\right)}, \quad t \in\left[t_{n}, t_{n+1}\right], \quad-\frac{J}{2} \leq l \leq \frac{J}{2}-1 .
$$

Combining (3.8) with (3.7) gives the solution to (3.4).

In practice, we use the second-order Strang splitting method [44] to couple (3.3) and (3.4), i.e., from $t=t_{n}$ to $t=t_{n+1}$, we solve

$$
\begin{aligned}
& \psi_{j}^{(1)}=\psi_{j}^{n} e^{-i \tau\left[V\left(x_{j}\right)+\beta\left|\psi_{j}^{n}\right|^{2 \sigma}\right] / 2}, \\
& \psi_{j}^{(2)}=\sum_{l=-J / 2}^{J / 2-1}\left(\widehat{\psi}_{l}^{(1)} e^{-i c \tau\left|\mu_{l}\right|^{2 \alpha}}\right) e^{i \mu_{l}\left(x_{j}-a\right)}, \quad 0 \leq j \leq J, \quad n \geq 0, \\
& \psi_{j}^{n+1}=\psi_{j}^{(2)} e^{-i \tau\left[V\left(x_{j}\right)+\beta\left|\psi_{j}^{(2)}\right|^{2 \sigma}\right] / 2},
\end{aligned}
$$

where $\psi_{j}^{n}$ denotes the numerical approximation of $\psi\left(x_{j}, t_{n}\right)$. When $n=0$, we have

$$
\psi_{j}^{0}=\psi_{0}\left(x_{j}\right), \quad 0 \leq j \leq J .
$$

For more general discussions on the time-splitting method, we refer readers to $[44,45]$ and references therein. Our method has spectral-order accuracy in space and second-order accuracy in time. It is also explicit, making it easy to implement via the fast Fourier transform (FFT). The memory cost is $O(J)$ and the computational cost per time step is $O(J \ln J)$ for 1D cases. In $2 \mathrm{D}$ (resp. $3 \mathrm{D}$ ), the memory cost is $O(J K)$ (resp. $O(J K L)$ ), and the computational cost per time step is $O(J K \ln (J K))$ (resp. $O(J K L \ln (J K L)))$, where $K$ and $L$ represent the number of intervals in $y$ and $z$-direction, respectively.

\section{Dynamics of the fractional Schrödinger equation}

We numerically study the dynamics of the 1D fractional Schrödinger equation with harmonic potential $V(x)=x^{2} / 2$, and remarkably, we see decoherence and emergence of high frequencies in the linear case. We choose the initial condition as a translation of the ground state $\psi_{g}$ :

$$
\psi_{0}(x)=\psi_{g}\left(x-x_{0}\right), \quad x \in \mathbb{R} .
$$

It can be viewed as a perturbation of the ground state: the larger the value of $x_{0}$, the stronger the perturbation. The ground state $\psi_{g}(x)$ is computed numerically with the same parameters $\alpha$ and $\beta$ used in the dynamics.

We have verified that our numerical results are invariant as decreasing the mesh size $h$ and time step $\tau$. Additionally, we make sure that the mass of the wave function (1.4) and the total energy (1.5) are conserved in our simulations. 


\subsection{Ground states}

To prepare for the dynamics, we first present the ground states of the Schrödinger equation. Figure 3 shows the modulus of the ground state $\left|\psi_{g}(x)\right|$ for different values of the parameters $\alpha$ and $\beta$, computed using the numerical methods proposed in [46]. The nonlocal effect from the fractional

(a)

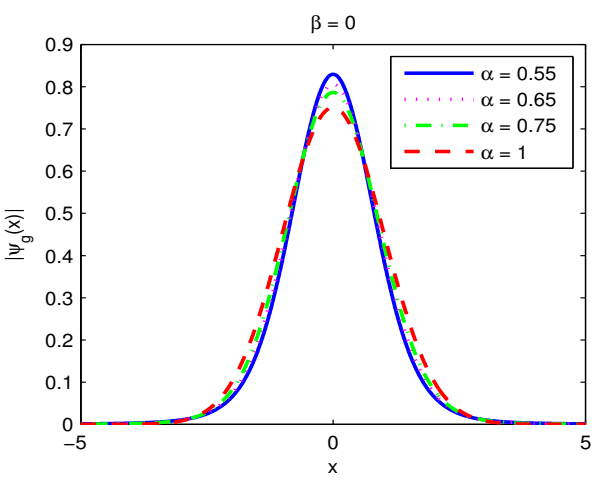

(b)

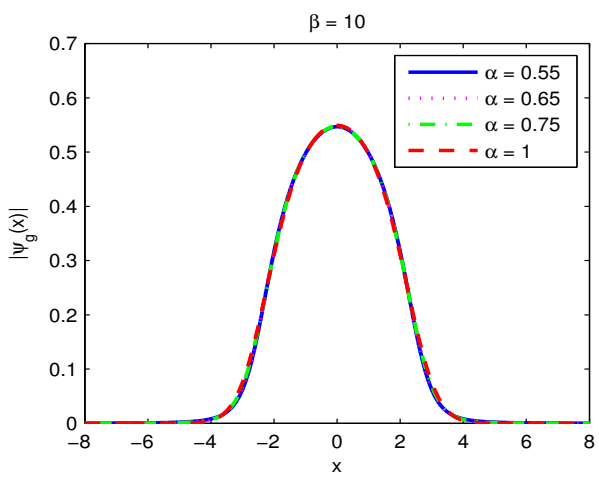

Figure 3: Ground states of the Schrödinger equation (1.1) with harmonic potential $V(x)=\frac{1}{2} x^{2}$. (a) $\beta=0$ (where nonlocal effects are significant); (b) $\beta=10$.

Laplacian $(-\Delta)^{\alpha}$ is significant when $\beta=0$ : the ground states are narrower and taller for smaller $\alpha$ (see Fig. 3(a)). By contrast, the local nonlinear interactions dominate when $\beta$ is large: the ground states are almost the same for different $\alpha$ (see Fig. 3(b)).

Remark 4.1. In work of Amore et al. [35], collocation methods based on little sinc functions were introduced to compute the ground states of the fractional linear Schrödinger equation:

$$
i \partial_{t} \psi(x, t)=(-\Delta)^{\gamma / 2} \psi(x, t)+x^{2} \psi(x, t), \quad x \in \mathbb{R} ; \quad\|\psi(\cdot, t)\|=1 .
$$

When $\gamma=2$, (4.2) reduces to the standard linear Schrödinger equation, and its ground state can
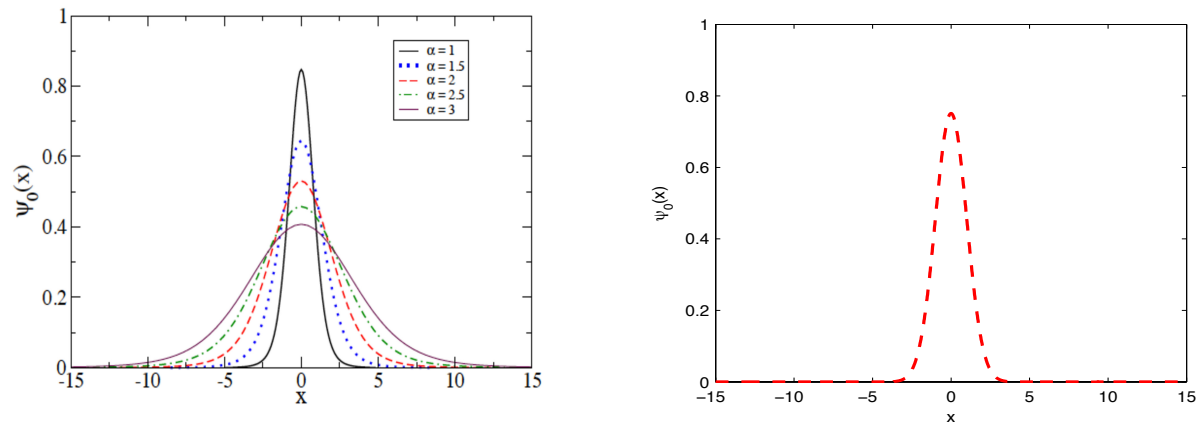

Figure 4: Left: Ground states of Amore et al. [35], where $\alpha=\gamma$ is twice as large as our $\alpha$. Right: The exact ground state (4.3) of standard Schrödinger equation which should correspond to the middle red dashed line in the left panel for $\alpha=2$. To be consistent with the notations in [35], the modulus of ground states is represented by $\psi_{0}(x)=\left|\psi_{g}(x)\right|$.

be found exactly with constant $\theta \in \mathbb{R}$ :

$$
\psi_{g}(x)=\frac{1}{\pi^{1 / 4}} e^{-\frac{x^{2}}{2}} e^{i \theta}, \quad x \in \mathbb{R} .
$$


However, the ground state of the standard Schrödinger equation in Figure 1 of the literature [35] (or see our Fig. 4 (left) with $\alpha=2$ ) is not consistent with the exact solution in (4.3), which may imply that the numerical method proposed in [35] for computing ground states is incorrect.

\subsection{Decoherent dynamics in linear cases}

We numerically study the dynamics of the 1D fractional linear $(\beta=0)$ Schrödinger equation, considering different initial translations $x_{0}$ in (4.1), or equivalently by ground state symmetry, different initial centers of mass $\langle X\rangle(0)=x_{0}$. Figures 5 and 6 show the time evolution of the solution $|\psi(x, t)|$ for various $\langle X\rangle(0)$ and $\alpha=0.75$ or $\alpha=1$. We display the results in a small
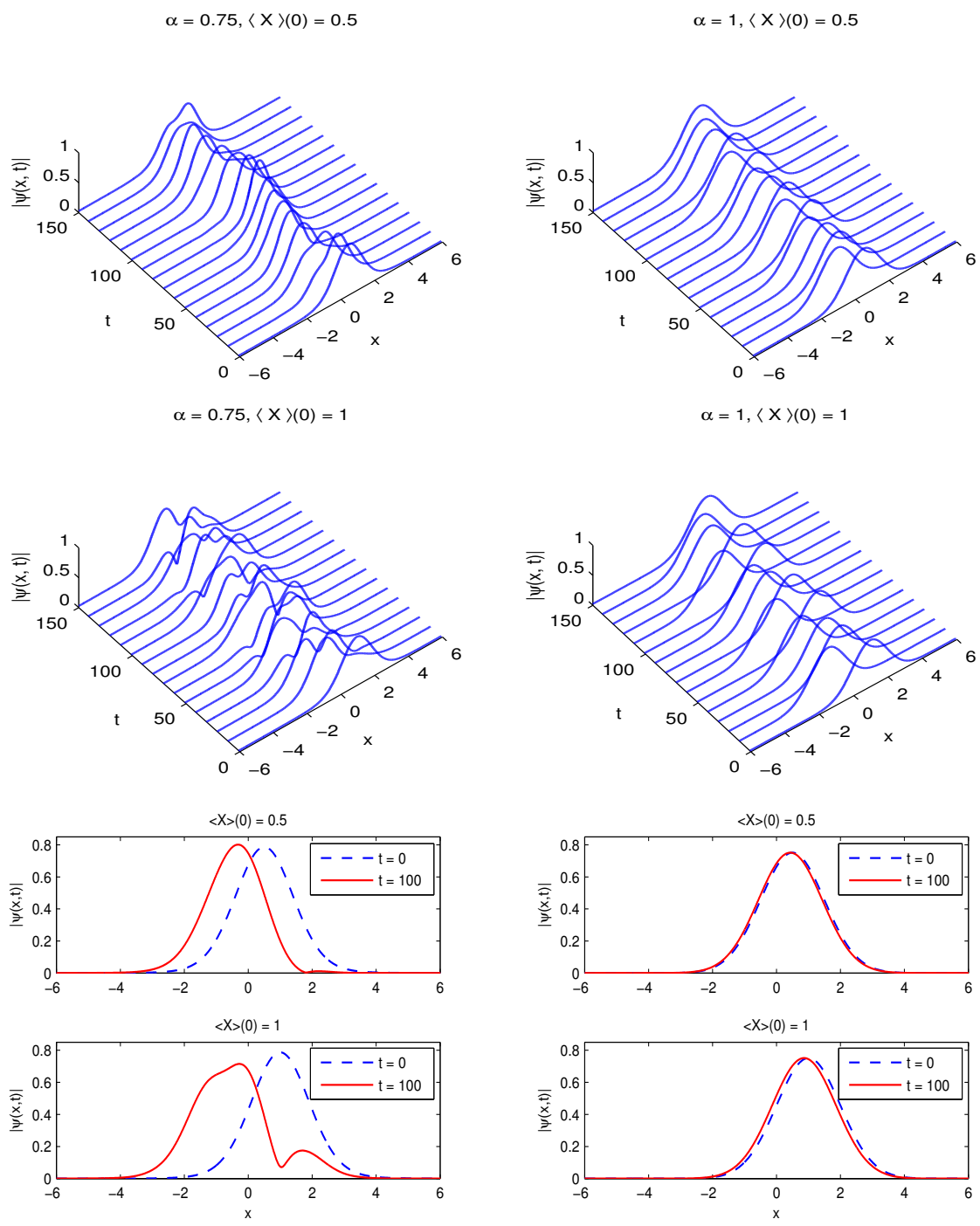

Figure 5: Time evolution of the solution of the fractional (left) and standard (right) linear Schrödinger equation (1.1) with relatively small shifts of the initial state (4.1). Decoherence emerges in the fractional linear case once the initial shift is large enough, as in the case of $\langle X\rangle(0)=1$.

region, although our computational domain is much larger.

For reference, the non-fractional case with $\alpha=1$ appears in the right panel of Figs. 5 and 6 , with the wave function oscillating around the trap center for $t>0$, retaining its initial ground 
state profile independent of the initial center of mass $\langle X\rangle(0)$, showing coherence, consistent with previous results $[14,15,25,26,47]$. By contrast, for $\alpha<1$ in the left panel of Figs. 5 and 6 , the
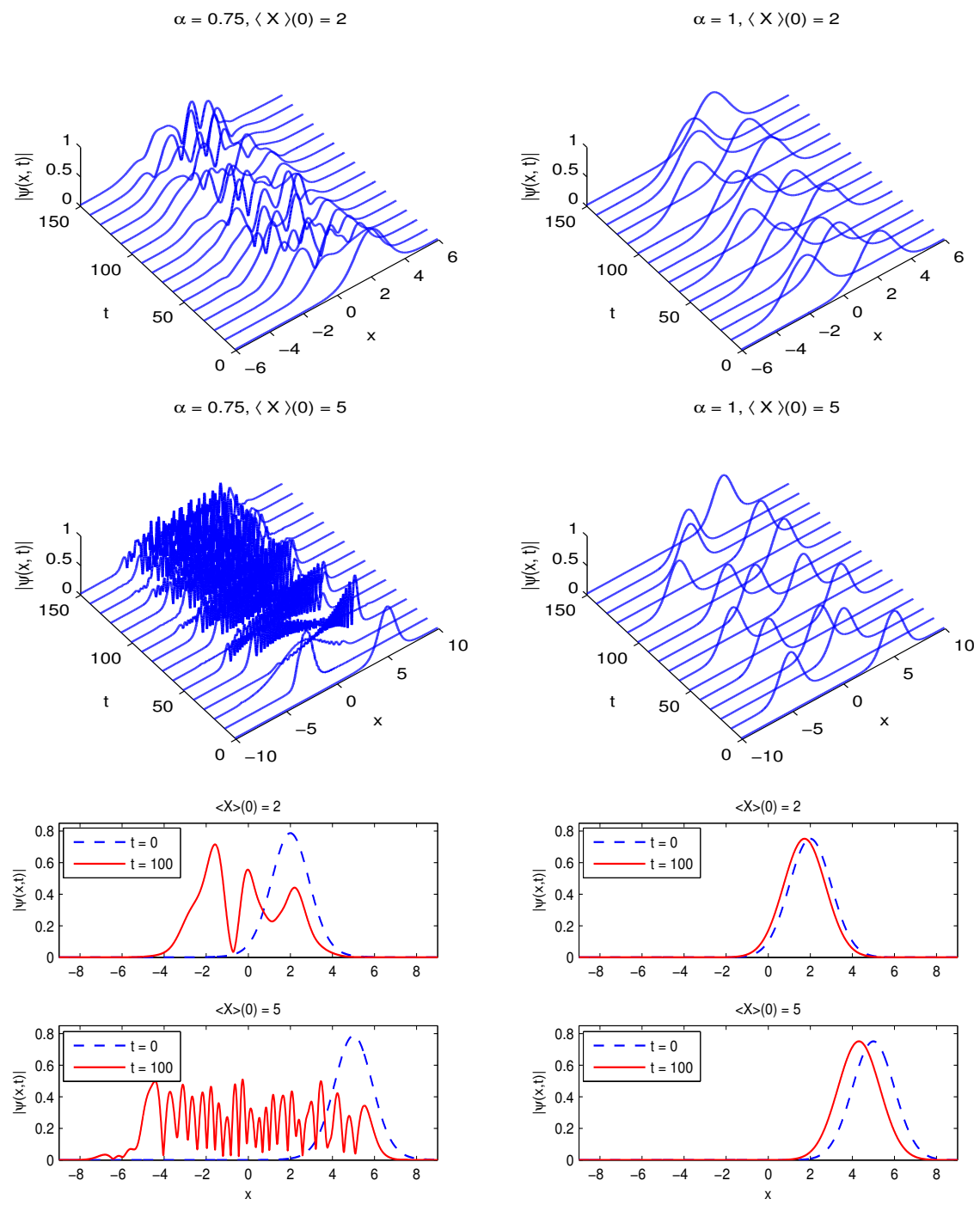

Figure 6: Time evolution of the solution of the fractional (left) and standard (right) linear Schrödinger equation (1.1) with larger shifts of the initial state (4.1). Decoherence is clear in the fractional case with initial shift $\langle X\rangle(0)=2$ (upper left) and high frequencies emerge with larger shift $\langle X\rangle(0)=5$ (middle left).

dynamics of the solution depends crucially on the initial center of mass $\langle X\rangle(0)$. For relatively small initial center of mass $\langle X\rangle(0)$ as in Fig. 5, the solution oscillates around the trap center, and its shape changes slightly. For larger $\langle X\rangle(0)$ as in Fig. 6, the solution changes dramatically during the dynamics, and the initial profile is completely destroyed after some time. Comparing Figs. 5 and 6 , we find that the larger the initial center of mass $\langle X\rangle(0)$, the stronger the decoherence of the solution.

To further study the decoherence as the solution evolves, we introduce two quantities: one is

$$
M(t):=\left(\int_{\mathbb{R}^{d}}\left(\left|\psi_{g}(\mathbf{x}-\langle X\rangle(t))\right|-|\psi(\mathbf{x}, t)|\right)^{2} d \mathbf{x}\right)^{1 / 2}, \quad t \geq 0,
$$


where $\psi_{g}$ is the ground states as in (4.1), that is, $M(t)$ measures the difference in shape of the solution at time $t$ from its initial condition. If the solution at time $t$ retains its initial profile, then $M(t)=0$. The other quantity is the variance

$$
S(t):=\int_{\mathbb{R}^{d}}[\mathbf{x}-\langle X\rangle(t)]^{2}|\psi(\mathbf{x}, t)|^{2} d \mathbf{x}, \quad t \geq 0 .
$$

This measures the spread of the solution around its center of mass, estimating the square width of the wave function [48]: If the shape of the solution does not change over time, then $S(t)$ remains a constant. Figure 7 displays the time evolution of $M(t)$ and $S(t)-S(0)$ for various $\alpha$ and $\langle X\rangle(0)$.
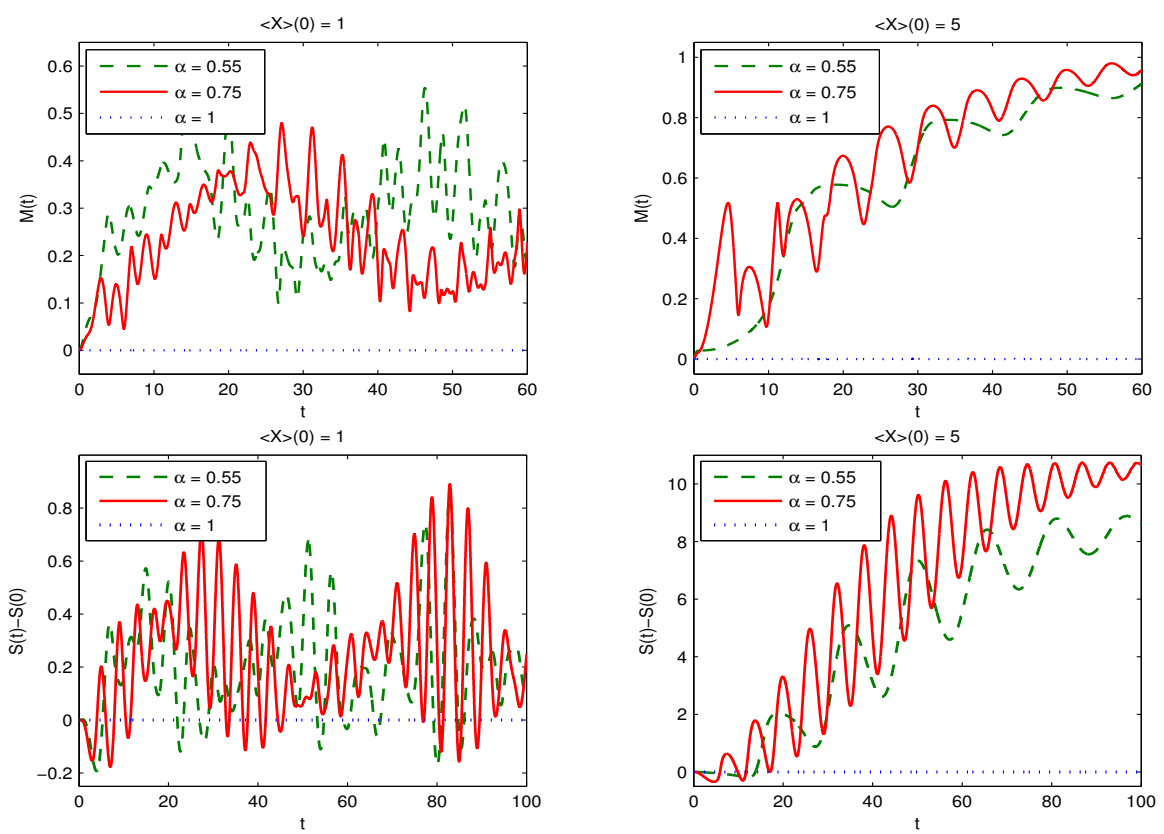

Figure 7: Time evolution of $M(t)$ (top) and $S(t)-S(0)$ (bottom) for measuring the decoherence in the 1D linear Schrödinger equation. Note that the coherence of the non-fractional case $\alpha=1$ is reflected in the dotted lines that are zeros for $M(t)$ and $S(t)-S(0)$.

For the standard NLS with $\alpha=1$, we find $M(t) \equiv 0$ and $S(t) \equiv S(0)$, for any $\langle X\rangle(0)$, which implies that the solution retains its initial shape during the dynamics. If $\alpha<1$, the $M(t)$ increases after a short time, implying that the solution distorts quickly in the fractional cases. Furthermore, the time evolution of $S(t)-S(0)$ shows that for fixed $\alpha$, the larger the initial center of mass $\langle X\rangle(0)$, the stronger the decoherence, which is consistent with our observations of density plots in Figs. 5 and 6 .

We conjecture that for $t \geq t_{0}$ large enough, the paths of both $M(t)$ and $|S(t)-S(0)|$ are bounded away from zero if $\langle X\rangle(0)$ is sufficiently large. We find that the solution of the standard NLS behaves more like a "particle," but the solution of the fractional NLS behaves more like a wave with effects that might be described as "interference" arising from the long-range interactions of the fractional Laplacian. This is the main difference between the standard and fractional NLS dynamics.

The expected position and momentum observables $\left(\langle X\rangle,\left\langle P_{\alpha}\right\rangle\right)$ reflect the decoherence, albeit in a subtle way: For $\alpha=1$, the trajectory of $(\langle X\rangle,\langle P\rangle)$ is a circle of radius $|\langle X\rangle(0)|$, which is consistent with the analytical solution of (2.7)-(2.8). When $\frac{1}{2}<\alpha<1$, the dynamics of $\left(\langle X\rangle,\left\langle P_{\alpha}\right\rangle\right)$ depends on the initial center of mass $\langle X\rangle(0)$ (see Fig. 8). Note that due to the initial setup in (4.1), the 
initial expected momentum $\left\langle P_{\alpha}\right\rangle(0)$ vanishes for any $\alpha$ and $\langle X\rangle(0)$. There are different regimes of
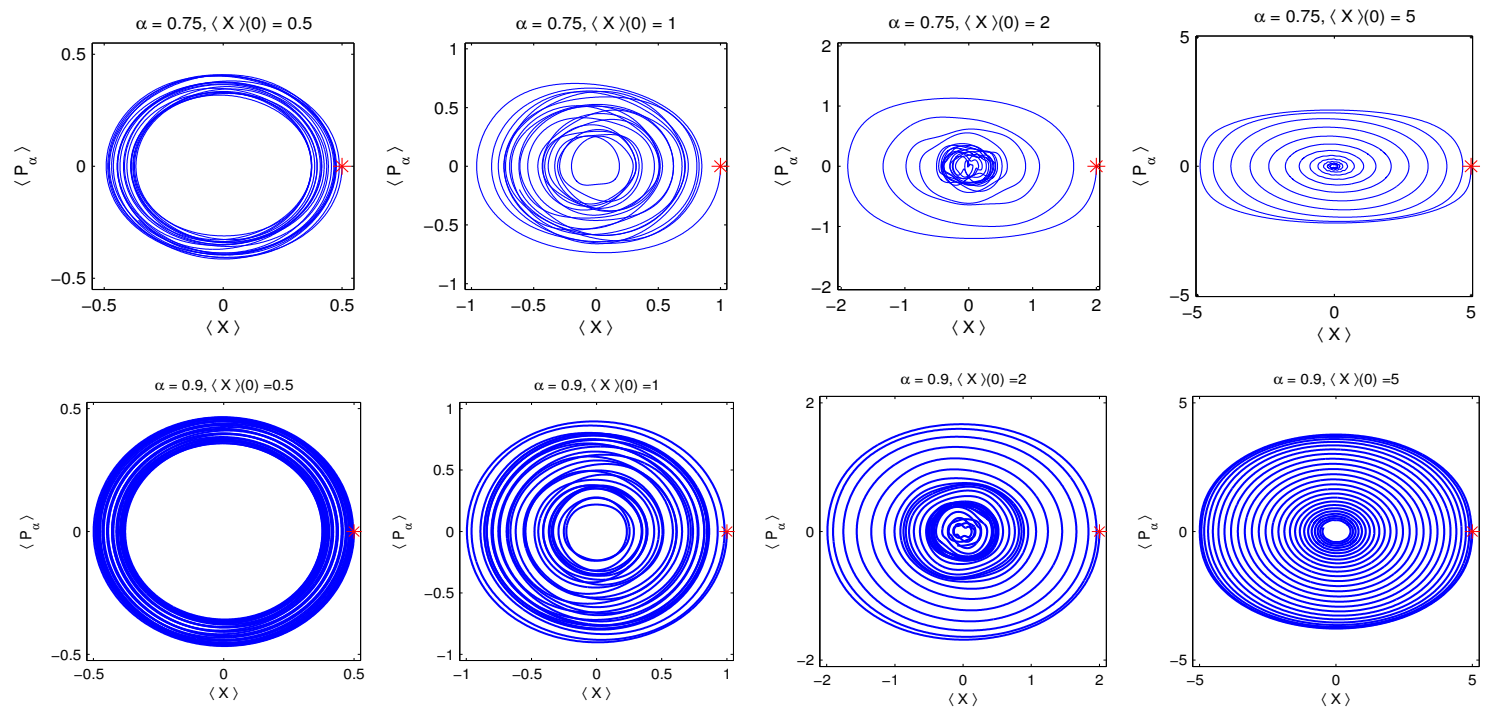

Figure 8: Trajectory of $\left(\langle X\rangle,\left\langle P_{\alpha}\right\rangle\right)$ for time $t \in[0,200]$ in the fractional linear Schrödinger equation (1.1) with initial condition (4.1). A red asterisk represents the initial expectations.

behavior, depending on the size of $|\langle X\rangle(0)|$. For small initial center of mass $|\langle X\rangle(0)|$, the trajectory of $\left(\langle X\rangle,\left\langle P_{\alpha}\right\rangle\right)$ goes towards an elliptic attractor; for larger $|\langle X\rangle(0)|$, the attractor is smaller, and if $|\langle X\rangle(0)|$ is large enough, the oscillations of these expected values are damped out over time, and the trajectory spirals towards $(0,0)^{T}$.

In addition, similar to $[49,50]$, we define the center of mass of the wave function $\psi$ (which could be thought of as the expectation of a "complex probability measure"):

$$
Z(t):=\int_{\mathbb{R}} x \psi(x, t) d x, \quad t \geq 0,
$$

and show the dynamics of $(\operatorname{Re}(Z), \operatorname{Im}(Z))$ for various $\alpha$ in Figure 9. For the standard NLS with $\alpha=1$, the evolution of $(\operatorname{Re}(Z), \operatorname{Im}(Z))$ is periodic, implying the recurrence of the initial state during the dynamics (see Fig. 9 bottom row). In the fractional case, the dynamics of $(\operatorname{Re}(Z), \operatorname{Im}(Z))$ becomes asymmetric around the origin (see Fig. 9 top and middle rows) and is not periodic, in contrast with the standard case (see Fig. 9 bottom row). These results are similar to those observed in $[49,50]$ for the emergence of chaos in the discrete Schrödinger equation with long-range interactions. Our results suggest that the decoherence comes from the long-range interactions due to the fractional Laplacian rather than the nonlinearity.

\subsection{Reduced decoherence in nonlinear cases}

We study the dynamics of 1D fractional NLS with local (or short-range) interactions from $\beta \neq 0$ in (1.1). Figures 10 and 11 show the time evolution of the solution $|\psi(x, t)|$ for $\alpha=0.75$ or $\alpha=0.9$. Here we omit the results for $\alpha=1$, as they are similar to those in Figs. 5 and 6 for linear cases.

For the fractional NLS, the dynamics of the solution depends on the fractional power $\alpha$, the nonlinear parameter $\beta$, and the initial center of mass $\langle X\rangle(0)$. For fixed $\alpha$ and $\beta$, if $|\langle X\rangle(0)|$ is small, the profile of the solution changes slightly during the dynamics, but almost no decoherence is observed, especially when $\beta$ is large (see Fig. 11 top row). If $|\langle X\rangle(0)|$ is large, however, weak 

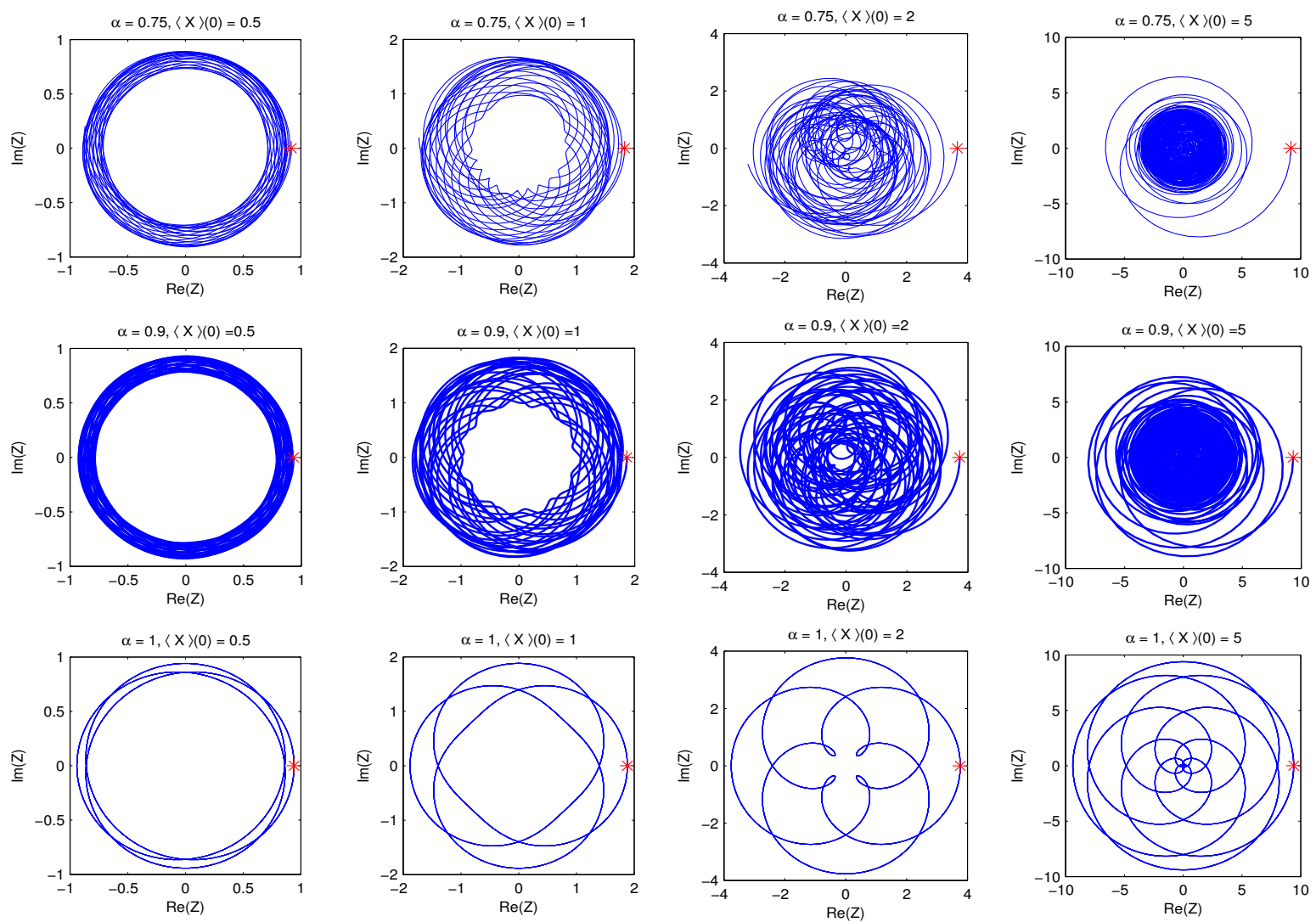

Figure 9: Trajectory of $(\operatorname{Re}(Z), \operatorname{Im}(Z))$ for time $t \in[0,100]$ in fractional linear Schrödinger equation (1.1) with initial condition (4.1). A red asterisk represents the initial condition.

decoherence might appear after some time, depending on the competition between the local nonlinear interactions and the nonlocal interactions from fractional Laplacian $(-\Delta)^{\alpha / 2}$. For instance, when $\alpha=0.9$ and $\langle X\rangle(0)=5$, decoherence emerges around $t=40$ if $\beta=1$, and it remains weak during the dynamics. But for the same $\alpha$ and initial offset, almost no decoherence is observed until $t=150$ if $\beta=10$, implying that strong local nonlinear interactions might suppress the decoherence especially when $\alpha$ is close to 1 . Comparing the results of $\alpha=0.75$ in Fig. 10 and Figs. 5-6, we find that even weak local nonlinear interactions can significantly reduce the decoherence of the solution in the fractional NLS dynamics. For the same $\beta$ and $\langle X\rangle(0)$, the smaller the power $\alpha$, the stronger the nonlocal interactions, the stronger the decoherence.

To further understand the nonlinear effects, the time evolution of $M(t)$ is presented in Figure 12 for $\beta=1$ and 10. We find that: (i) For the same $\alpha$ and $|\langle X\rangle(0)|$, the value of $M(t)$ decreases when increasing the nonlinear parameter $\beta$, which implies that the local nonlinear interactions prevent the loss of soliton during the dynamics. (ii) For the same $\beta$ and $|\langle X\rangle(0)|$, the value of $M(t)$ is generally larger when the fractional power $\alpha$ is smaller, indicating the change in the shape of solution is mainly caused by the nonlocal interactions from the fractional Laplacian $(-\Delta)^{\alpha / 2}$.

The dynamics of $S(t)-S(0)$ in Figure 13 shows the similar phenomena, where the coherence of the standard NLS $(\alpha=1)$ is reflected by $S(t) \equiv S(0)$ for any $t \geq 0$. In the fractional case $\frac{1}{2}<\alpha<1, S(t)-S(0)$ oscillates around zero. When $\alpha$ is far from $\frac{1}{2}$, the oscillation of $S(t)-S(0)$ generally reduces as $\beta$ increases, which implies that the spread of the solution around its center of mass decreases with strong local nonlinear interactions. However, when $\alpha$ is near the lower end of 
$\beta=1, \alpha=0.75,<X>(0)=1$

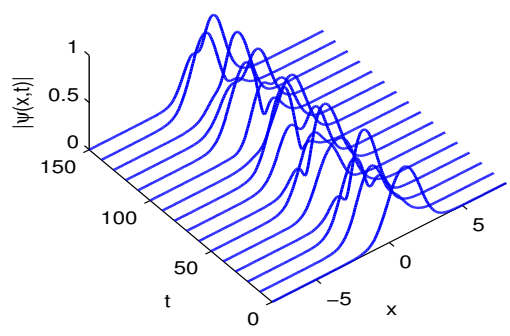

$\beta=1, \alpha=0.75,<X>(0)=5$
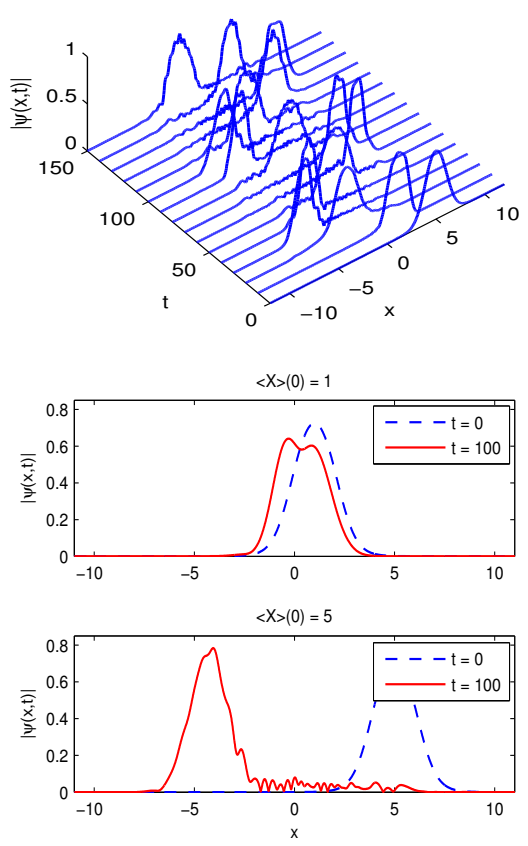

$\beta=1, \alpha=0.9,<X>(0)=1$

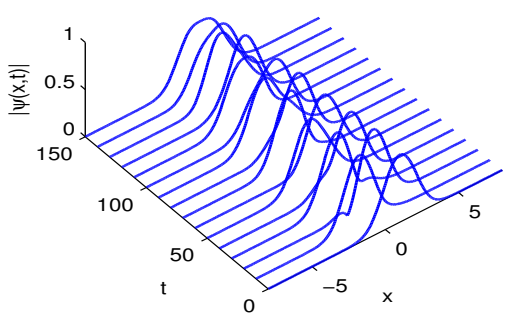

$\beta=1, \alpha=0.9,<X>(0)=5$
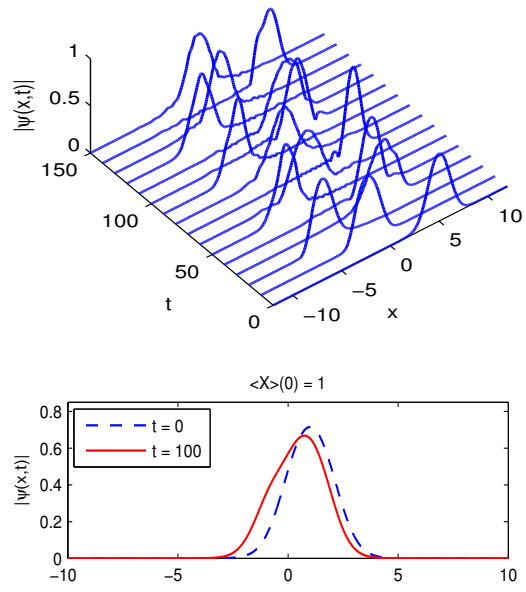

$<X>(0)=5$

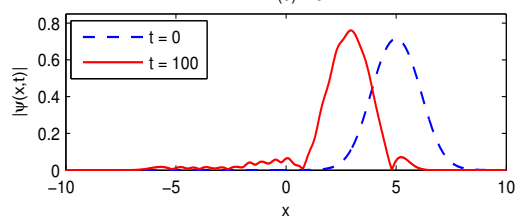

Figure 10: Time evolution of the solution of the fractional NLS (1.1) with weak nonlinear interactions (i.e., small $\beta$ ). Decoherence is reduced due to local nonlinear interactions.

the range $\left(\frac{1}{2}, 1\right]$ and $|\langle X\rangle(0)|$ is larger, the strong local nonlinear interactions could introduce large fluctuations that increase the spread of solution $S(t)$ (see Fig. 13 bottom right).

Figure 14 shows the trajectories of $\left(\langle X\rangle,\left\langle P_{\alpha}\right\rangle\right)$ for $\alpha=0.75$, with the effect of local nonlinear interactions in the fractional NLS. Note that when $\alpha=1$, the trajectory of $(\langle X\rangle,\langle P\rangle)$ is a circle of radius of $|\langle X\rangle(0)|$, independent of nonlinear parameter $\beta$. Comparing Figs. 8 (upper row) and 14, we find that the local nonlinear interactions have a strong effect on the dynamics of $\left(\langle X\rangle,\left\langle P_{\alpha}\right\rangle\right)$, which prevent the decay of the expected position and momentum. For example, when $\alpha=0.75$ and $\langle X\rangle(0)=5$, the trajectory is a spiral in the linear case (see Fig. 8 upper right), implying that $\langle X\rangle(t)$ and $\left\langle P_{\alpha}\right\rangle(t)$ decay over time, however, it appears to move towards an attractor when $\beta \neq 0$ (see Fig. 14 right column). This nonlinear effect can be also observed in the time evolution of $\langle X\rangle(t)$ and $\left\langle P_{\alpha}\right\rangle(t)$ in Fig. 2. Additionally, our extensive simulations show that when $\beta$ is large and $\alpha$ is far from $\frac{1}{2}$, the dynamics of $\left(\langle X\rangle,\left\langle P_{\alpha}\right\rangle\right)$ becomes less sensitive to $\beta$, and the trajectories of $\left(\langle X\rangle,\left\langle P_{\alpha}\right\rangle\right)$ are similar to those in Fig. 14 (lower row). 
$\beta=10, \alpha=0.75,<X>(0)=1$

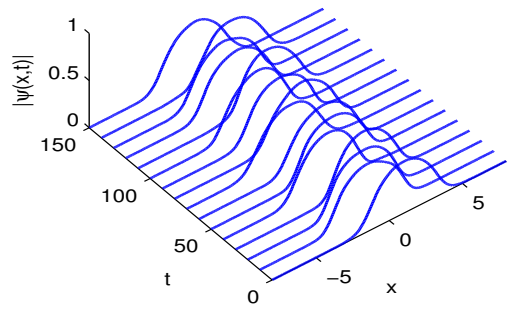

$\beta=10, \alpha=0.75,<X>(0)=5$
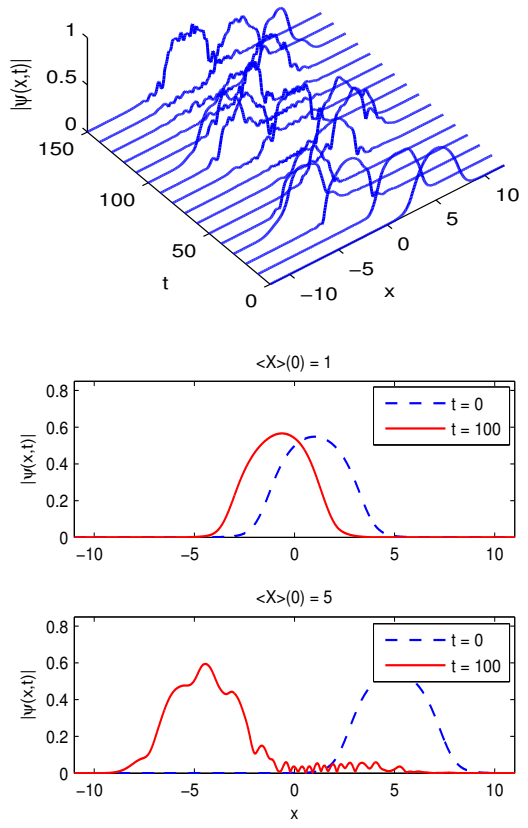

$\beta=10, \alpha=0.9,<X>(0)=1$

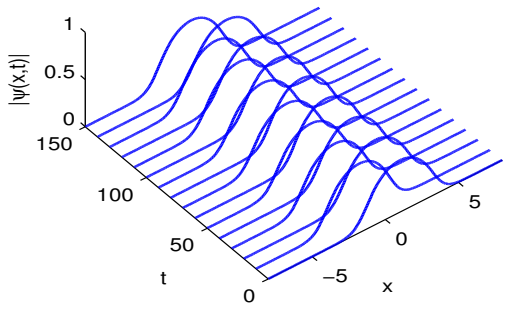

$\beta=10, \alpha=0.9,<X>(0)=5$
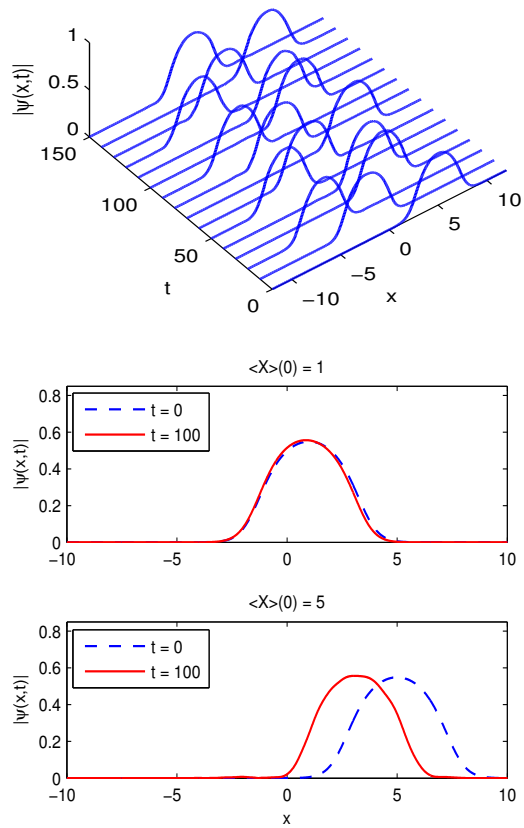

Figure 11: Time evolution of the solution of the fractional NLS (1.1) with strong nonlinear interactions (i.e., large $\beta$ ). Decoherence is reduced due to nonlinear interactions.

In Figures 15 and 16, we present the dynamics of $(\operatorname{Re}(Z), \operatorname{Im}(Z))$ for various $\alpha$ and $\beta$, which shows that including local nonlinear interactions significantly affects the dynamics of the solution. When $\alpha=1$, the phase diagrams $(\operatorname{Re}(Z), \operatorname{Im}(Z))$ in Fig. 15 are completely different from the linear case in Fig. 9, even though the trajectories of $(\langle X\rangle,\langle P\rangle)$ are the same for both linear and nonlinear cases. In the fractional cases with $\frac{1}{2}<\alpha<1$, the dynamics of $(\operatorname{Re}(Z), \operatorname{Im}(Z))$ again reflect the effects of nonlinear interactions. When $\beta$ is large, the time evolution of $(\operatorname{Re}(Z), \operatorname{Im}(Z))$ in the fractional cases become closer to that in the non-fractional case (see the lower right diagrams in Fig. 16, which are more like those in Fig. 15). The stronger nonlinearity appears to reduce the decoherence effect of the fractional Laplacian $(-\Delta)^{\alpha / 2}$.

Remark 4.2. In Sections 4.2 and 4.3, the initial condition $\psi_{0}(\mathbf{x})$ is chosen as a translation of the ground state $\psi_{g}$ in (4.1), and decoherence is observed in dynamics of fractional Schrödinger equation. In fact, similar decoherence structure is also observed with other initial profiles. Hence, we conclude that the emergence of the decoherence is dependent on the fractional Laplacian $(-\Delta)^{\alpha / 2}$, 

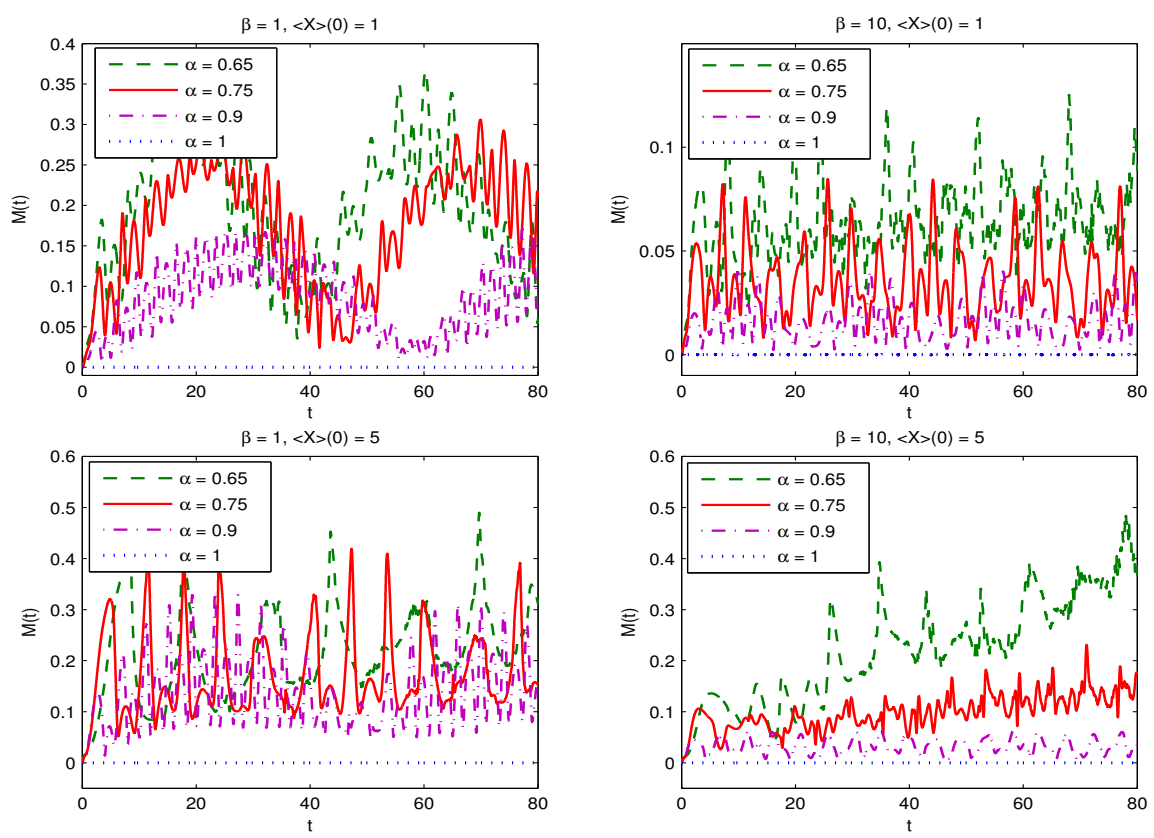

Figure 12: Time evolution of $M(t)$ for studying the decoherence in the 1D NLS.
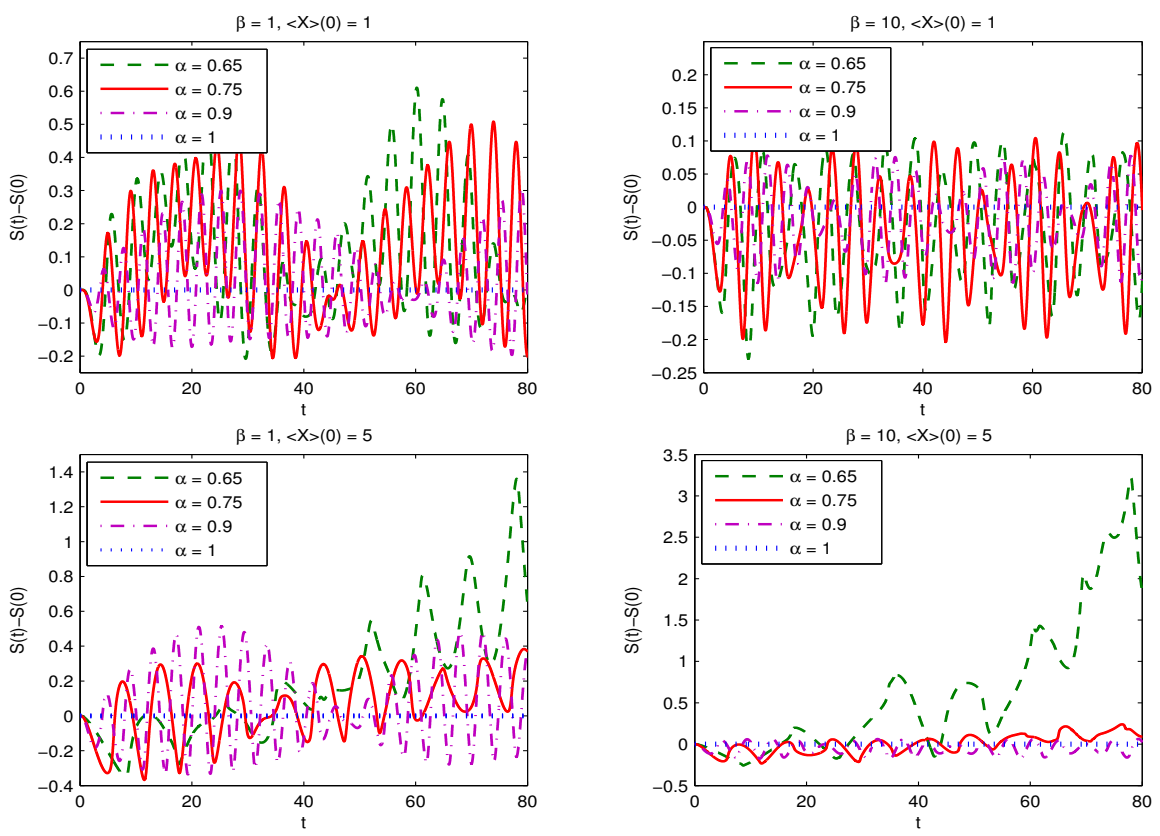

Figure 13: Time evolution of $S(t)-S(0)$ for studying the decoherence in the 1D NLS.

but largely independent of the initial profile $\psi_{g}$.

\section{Discussion and conclusion}

We studied the solution dynamics of the fractional Schödinger equation with a harmonic potential. First, we obtained Newtonian equations of motion for the expectations of the position and momen- 

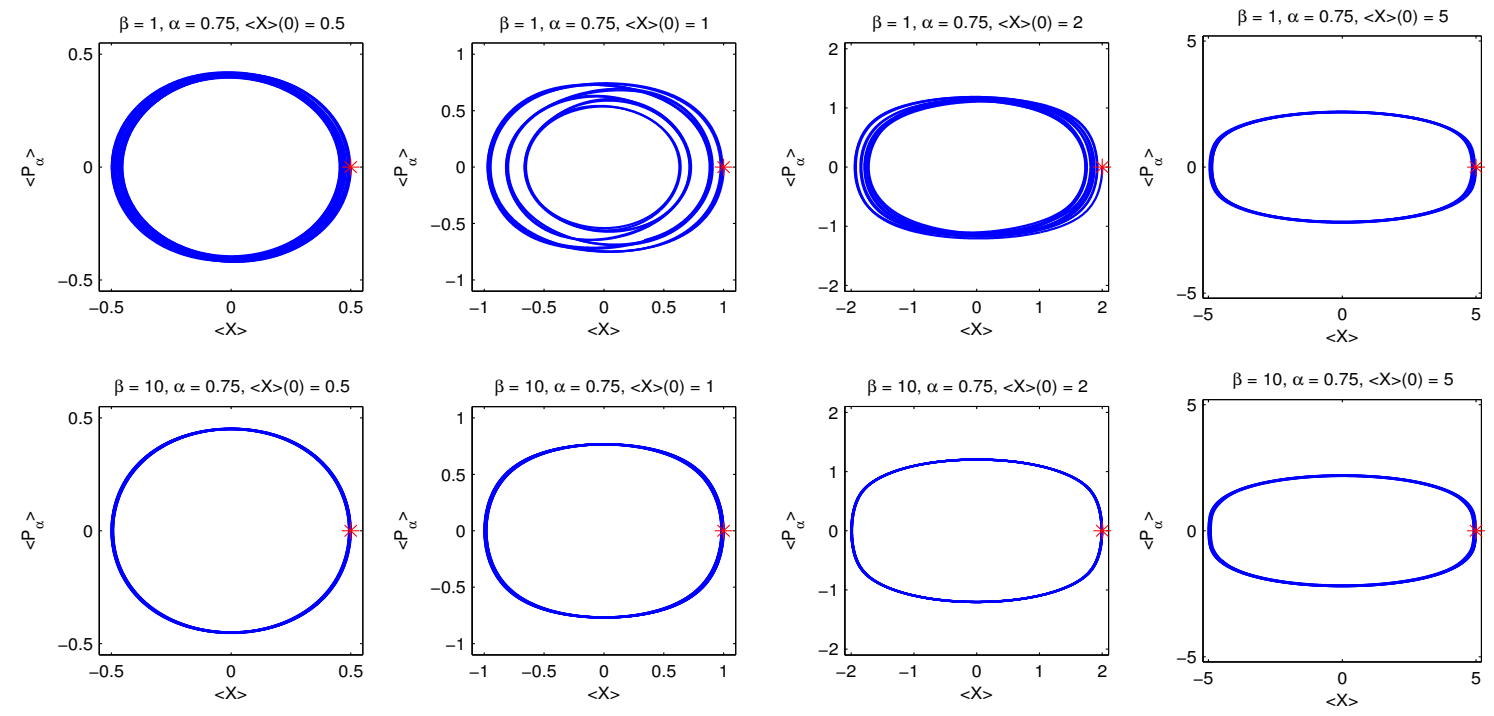

Figure 14: Trajectory of $\left(\langle X\rangle,\left\langle P_{\alpha}\right\rangle\right)$ for time $t \in[0,100]$ in the fractional NLS (1.1) with initial condition (4.1). A red asterisk represents the initial expectations.
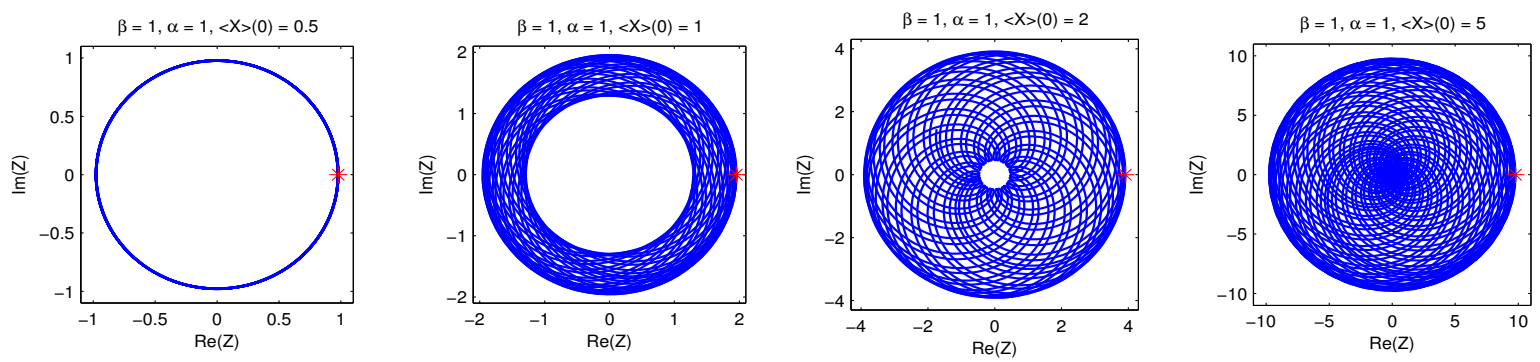

Figure 15: Trajectories of $(\operatorname{Re}(Z), \operatorname{Im}(Z))$ for time $t \in[0,100]$ in the standard NLS (1.1) with initial condition (4.1) represented by a red asterisk. The standard NLS has periodic trajectories.

tum observables in the fractional Schrödinger equation, solving an open problem in the study of soliton dynamics [9]. These equations are not closed in the fractional case, unlike the corresponding equations for the standard Schrödinger equations.

Second, we numerically simulated the dynamics of the fractional Schrödinger equation with a soliton as the initial condition. In the standard Schrödinger dynamics, no decoherence was observed, consistent with results in the literature. The decoherence emerges in the fractional Schrödinger dynamics, however, it remains weak if the initial center of mass (expected position) is small. If the initial center of mass is large, the decoherence could become very strong when the fractional power is small. Our results are consistent with those observed in the study of the discrete Schrödinger equation with long-range interactions [49-51]. When a nonlinearity is added to the fractional Schrödinger dynamics, decoherence and fluctuations are observed if the initial center of mass is large. Compared to the results in linear cases, we found that the local nonlinear interactions can reduce or delay the emergence of decoherence in the dynamics. Our results suggest that the decoherence comes from the long-range interactions due to the fractional Laplacian rather than the nonlinearity. More work is needed to better understand this decoherence in the soliton dynamics of fractional Schrödinger equations. 

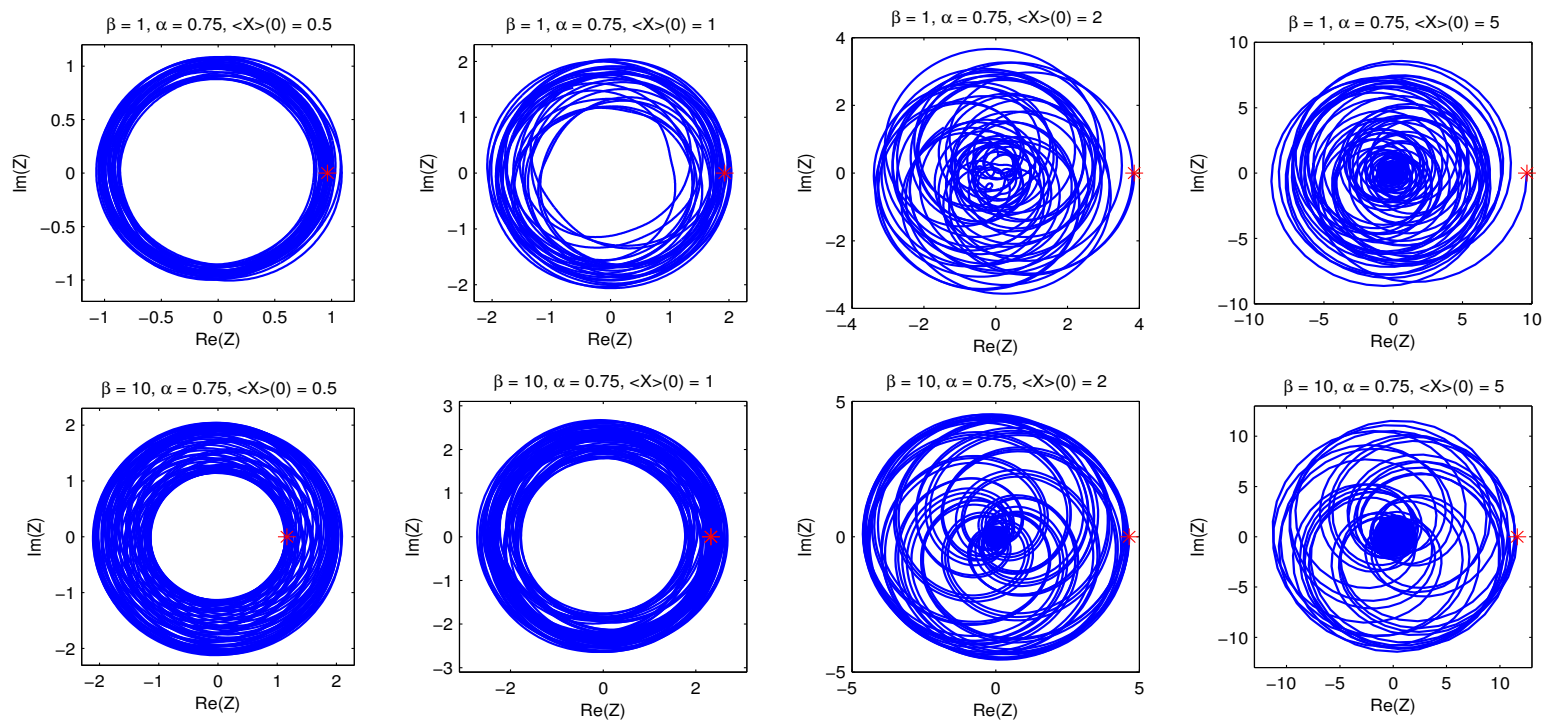

Figure 16: Trajectory of $(\operatorname{Re}(Z), \operatorname{Im}(Z))$ for time $t \in[0,100]$ in the fractional NLS (1.1) with initial condition (4.1) represented by a red asterisk. The stronger nonlinearity (bottom row) gives trajectories closer to the periodic trajectories of the traditional NLS in Fig. 15.

Acknowledgements. K.K. was partially supported by National Science Foundation grants OISE0730136, DMS-1106770, and CAREER DMS-1254791. Y.Z. was partially supported by National Science Foundation grant DMS-1217000, Simons Foundation Award No. 210138, and the University of Missouri Research Board. Many thanks to the anonymous reviewer.

\section{References}

[1] Y. B. Gaididei, S. F. Mingaleev, P. L. Christiansen and K. Ø. Rasmussen, Effects of nonlocal dispersive interactions on self-trapping excitations, Phys. Rev. E, 55 (1997), pp. 6141-6150.

[2] N. Laskin, Fractional Schrödinger equation, Phys. Rev. E, 66 (2002), 056108.

[3] A. Zoia, A. Rosso and M. Kardar, Fractional Laplacian in bounded domains, Phys. Rev. E, 76 (2007), 021116.

[4] T. M. Michelitsch, G. A. Maugin, A. F. Nowakowski, F. Nicolleau and M. Rahman, The fractional Laplacian as a limiting case of a self-similar spring model and application to $n$ dimensional anomalous diffusion, Fract. Calc. Appl. Anal., 16 (2013), pp. 827-859.

[5] S. Mingaleev, P. Christiansen, Y. Gaididei, M. Johannson and K. Rasmussen, Models for energy and charge transport and storage in biomolecules, J. Biol. Phys., 25 (1999), pp. 4163.

[6] J. Fröhlich, B.L.G. Jonsson and E. Lenzmann, Boson stars as solitary waves, Comm. Math. Phys., 274 (2007), pp. 1-30.

[7] E. Lenzmann, Well-posedness for semi-relativistic Hartree equations of critical type, Math. Phys. Anal. Geom., 10 (2007), pp. 43-64. 
[8] K. Kirkpatrick, E. Lenzmann and G. Staffilani, On the continuum limit for discrete NLS with long-range lattice interactions, Commun. Math. Phys., 317 (2013), pp. 563-591.

[9] S. Secchi and M. Squassina, Soliton dynamics for fractional Schrödinger equations, Appl. Anal., 93 (2014), pp. 1702-1729.

[10] Y. Hu and G. Kallianpur, Schrödinger equations with fractional Laplacians, Appl. Math. Optim., 42 (2000), pp. 281-290.

[11] S.G. Samko, A.A. Kilbas and O.I. Marichev, Fractional Integrals and Derivatives: Theory and Applications, CRC Press (1993).

[12] K. B. Oldham and J. Spanier, The Fractional Calculus: Theory and Applications of Differentiation and Integration to Arbitrary Order, Dover (2006).

[13] L. P. Pitaevskii and S. Stringari, Bose-Einstein Condensation, Oxford: Clarendon Press, 2003.

[14] W. Bao, Q. Du and Y. Zhang, Dynamics of rotating Bose-Einstein condensates and its efficient and accurate numerical computation, SIAM J. Appl. Math., 66 (2006), pp. 758-786.

[15] Y. Zhang and W. Bao, Dynamics of the center of mass in rotating Bose-Einstein condensates, Appl. Numer. Math., 57 (2007), pp. 697-709.

[16] R. L. Frank and E. Lenzmann, On the uniqueness and nondegeneracy of ground states of $(-\Delta)^{s} Q+Q-Q^{\alpha+1}=0$ in $\mathbb{R}$, Acta. Math., 210 (2013), pp. 261-318.

[17] R. L. Frank, E. Lenzmann and L. Silvestre, Uniqueness of radial solutions for the fractional Laplacian, Comm. Pure Appl. Math., (2015), doi:10.1002/cpa.21591.

[18] B. Guo and Z. Huo, Well-posedness for the nonlinear fractional Schrödinger equation and inviscid limit behavior of solution for the fractional Ginzburg-Landau equation, Fractional Calc. Appl. Anal., 16 (2013), pp. 226-242.

[19] B. Guo, Y. Sire, Y. Wang and L. Zhao, On the energy-critical fractional Schrödinger equation in the radial case, arXiv:1310.6816.

[20] Y. Cho, G. Hwang, S. Kwon and S. Lee, On the finite time blowup for mass-critical Hartree equations, P. Roy. Soc. Edinb. A, 145 (2015), pp. 467-479.

[21] H. Smith, Introduction to Quantum Mechanics, World Scientific Pub Co Inc, (1991), pp. $108-109$.

[22] J. Fröhlich, S. Gustafson, B.L.G. Jonsson and I. M. Sigal, Dynamics of solitary waves in an external potential, Comm. Math. Phys., 250 (2004), pp. 613-642.

[23] V. Benci, M.G. Ghimenti and A.M. Micheletti, The nonlinear Schrödinger equation: Soliton dynamics, J. Diff. Eqs., 249 (2010), pp. 3312-3341.

[24] J. Bronski and R. L. Jerrard, Soliton dynamics in a potential, Math. Res. Lett., 7 (2000), pp. $329-342$.

[25] J. J. García-Ripoll, V. M. Pérez-García and V. Vekslerchik, Construction of exact solutions by spatial translations in inhomogeneous nonlinear Schrödinger equations, Phys. Rev. E, 64 (2001), 056602. 
[26] Y. Zhang, Numerical study of vortex interactions in Bose-Einstein condensation, Commun. Comput. Phys., 8 (2010), pp. 327-350.

[27] M. Caliari and M. Squassina, Numerical computation of soliton dynamics for NLS equations in a driving potential, Electron. J. Diff. Eqns., 89 (2010), pp. 1-12.

[28] V. J. Ervin, N. Heuer and J. P. Roop, Numerical approximation of a time dependent, nonlinear, space-fractional diffusion equations, SIAM J. Numer. Anal., 45 (2007), pp. 572-591.

[29] M. M. Meerschaert, H.-P. Scheffler and C. Tadjeran, Finite difference methods for twodimensional fractional dispersion equation, J. Comput. Phys., 211 (2006), pp. 249-261.

[30] J.R. Roop, Computational aspects of FEM approximation of fractional advection dispersion equations on bounded domains in $\mathbb{R}^{2}$, J. Comput. Appl. Math., 193 (2006), pp. 243-268.

[31] D. Cordoba, F. Gancedo and R. Orive, A note on the interface dynamics for convection in porous media, Physica D., 237 (2008), pp. 1488-1497.

[32] L. Berselli, D. Cordoba and R. Granero-Belinchon, Local solvability and turning for the inhomogeneous Muskat problem, Interfaces and Free Boundaries, 16 (2014), pp. 175-213.

[33] R. Granero-Belinchón and R. Orive-Illera, An aggregation equation with a nonlocal flux, Nonlinear Anal.-Theor., 108 (2014), pp. 260-274.

[34] A. Bueno-Orovio, D. Kay and K. Burrage, Fourier spectral methods for fractional-in-space reaction-diffusion equations, BIT Numer. Math., 54 (2014), pp. 937-954.

[35] P. Amore, F. M. Fernández, C. P. Hofmann and R. A. Sáenz, Collocation method for fractional quantum mechanics, J. Math. Phys., 51 (2010), 122101.

[36] D. Cai, A. J. Majda, D. W. McLaughlin and E. G. Tabak, Dispersive wave turbulence in one dimension, Physica D, 152-153 (2001), pp. 551-572.

[37] A. J. Majda, D. W. McLaughlin and E. G. Tabak, A one-dimensional model for dispersive wave turbulence, J. Nonlinear Sci., 6 (1997), pp. 9-44.

[38] V. E. Zakharov, P. Guyenne, A. N. Pushkarev and F. Dias, Wave turbulence in onedimensional models, Physica D., 152-153 (2001), pp. 573-619.

[39] Q. Du, M. Gunzburger, R. B. Lehoucq and K. Zhou, Analysis and approximation of nonlocal diffusion problems with volume constraints, SIAM Review, 54 (2012), pp. 667-696.

[40] S. Duo and Y. Zhang, Computing the ground and first excited states of the fractional Schrödinger equation in an infinite potential well, Commun. Comput. Phys., 18 (2015), pp. $321-350$.

[41] D. Cordoba, F. Gancedo and R. Orive, Analytical behavior of 2D incompressible flow in porous media, J. Math. Phys., 48 (2007), 065206.

[42] R. Granero-Belinchón and J. Hunter, On a nonlocal analog of the Kuramoto-Sivashinsky equation, Nonlinearity, 28 (2015), pp. 1103-1133.

[43] S. Duo and Y. Zhang, Mass-conservative Fourier spectral methods for solving the fractional nonlinear Schrödinger equation, Comp. Math. Appl., (2016), doi:10.1016/j.camwa.2015.12.042. 
[44] G. Strang, On the construction and comparison of difference schemes, SIAM J. Numer. Anal., 5 (1968), pp. 506-517.

[45] G. Marchuk, Splitting and Alternating Direction Methods, in Handbook of Numerical Analysis, North-Holland, Amsterdam, 1990.

[46] K. Kirkpatrick and Y. Zhang, Ground states of fractional Schrödinger equation, in preparation.

[47] W. Bao, D. Marahrens, Q. Tang and Y. Zhang, A simple and efficient numerical method for computing the dynamics of rotating Bose-Einstein condensates via a rotating Lagrangian coordinate, SIAM J. Sci. Comput., 35 (2013), pp. A2671-A2695.

[48] C. Sulem and P.-L. Sulem, The nonlinear Schrödinger equation: Self-focusing and wave collapse, Springer-Verlag New York, 1999.

[49] G. M. Zaslavsky, M. Edelman and V. E. Tarasov, Dynamics of the chain of forced oscillators with long-range interaction: From synchronization to chaos, Chaos, 17 (2007), 043124.

[50] N. Korabel and G.M. Zaslavsky, Transition to chaos in discrete nonlinear Schrödinger equation with long-range interaction, Physica A, 378 (2007), pp. 223-237.

[51] V.E. Tarasov, Continuous limit of discrete systems with long-range interaction, J. Phys. A: Math. Gen., 39 (2006), pp. 14895-14910. 